\title{
A New AILC for a Class of Nonlinearly Parameterized Systems with Unknown Delays and Input Dead-Zone
}

\author{
Jian-ming Wei, Yun-an Hu, and Mei-mei Sun \\ Department of Control Engineering, Naval Aeronautical and Astronautical University, Yantai 264001, China \\ Correspondence should be addressed to Jian-ming Wei; wjm604@163.com
}

Received 23 March 2014; Revised 12 May 2014; Accepted 26 May 2014; Published 24 June 2014

Academic Editor: Bin Zhou

Copyright (C) 2014 Jian-ming Wei et al. This is an open access article distributed under the Creative Commons Attribution License, which permits unrestricted use, distribution, and reproduction in any medium, provided the original work is properly cited.

\begin{abstract}
This paper presents an adaptive iterative learning control (AILC) scheme for a class of nonlinear systems with unknown timevarying delays and unknown input dead-zone. A novel nonlinear form of deadzone nonlinearity is presented. The assumption of identical initial condition for ILC is removed by introducing boundary layer functions. The uncertainties with time-varying delays are compensated for with assistance of appropriate Lyapunov-Krasovskii functional and Young's inequality. The hyperbolic tangent function is employed to avoid the possible singularity problem. According to a property of hyperbolic tangent function, the system output is proved to converge to a small neighborhood of the desired trajectory by constructing Lyapunov-like composite energy function (CEF) in two cases, while maintaining all the closed-loop signals bounded. Finally, a simulation example is presented to verify the effectiveness of the proposed approach.
\end{abstract}

\section{Introduction}

Practically, many engineering systems carry out repetitive tasks in fixed finite space, such as manipulators [1-3]. In high precision engineering, perfect tracking for such tasks is highly desirable. Whereas existing control approaches, such as classical PID controllers, feedback linearization, and adaptive control, may guarantee closed-loop stability, they can hardly achieve perfect tracking. Fortunately, learning and repetitive control are the alternatives to address this problem, which enhance the tracking accuracy from operation to operation for systems executing repetitive tasks. By now, iterative learning control (ILC) has become one of the most important learning strategies owing to its implementation simplicity under the repeatable control environment. The basic idea of ILC is to improve the control performance of systems from trial to trial. Traditional iterative learning controllers have been developed for more than two decades for nonlinear plants [1-12]. The control input of traditional ILC is directly updated by a learning mechanism using the information of error and input in the previous iteration, and the contraction mapping theorem is often used to analyze the stability of closed-loop systems. However, the studied systems must satisfy global Lipschitz continuous condition. Thus, there are some difficulties or limitations to apply traditional ILC for certain systems. In order to circumvent this problem, some other new ILC algorithms have been widely studied. One of the most important developments is adaptive ILC (AILC) [13-15], in which the control parameters are adjusted between successive iterations, and the so-called composite energy function (CEF) [16] is usually constructed to derive the stability conclusions. In recent years, the control community has witnessed great progress in AILC of uncertain nonlinear systems [17-27].

In practical control tasks, systems with time delays are frequently encountered. The existence of time delays may make the controllers design more complicated and challenging, especially for systems with unknown time-delays. Stabilization problem of control systems with time delay has drawn much attention [28-35] due to its mathematical challenge and application demand in real-time control. In [28-31], the controller design and stability analysis for statedelayed systems were presented and [32-35] discussed the stabilization of systems with input delays. In the field of ILC, although so many results have been obtained, only few ones were available for time-delay nonlinear systems [36-41], and the majority of these works were traditional iterative learning 
controllers. In the framework of AILC, Chen and Zhang [40] proposed an AILC scheme for a class of scalar systems with unknown time-varying parameters and unknown timevarying delay. In [41], an adaptive learning control design was developed for a certain class of first-order nonlinearly parameterized systems with unknown periodically timevarying delay and further extended to a class of highorder systems with both time-varying and time-invariant parameters. However, they all required the identical initial conditions on the initial states and the reference trajectory for the AILC design, which is necessary for the stability and convergence analysis but can hardly be satisfied in practical systems.

In practice, nonsmooth and nonlinear characteristics such as dead-zone, hysteresis, saturation, and backlash are common in actuator and sensors. Dead-zone is one of the most important nonsmooth and non-affine-in-input nonlinearities in many industrial processes, which can severely deteriorate system performances and give rise to extra difficulties in the controller design. Therefore, the effect of dead-zone should be taken into consideration and has been drawing much interest in the control community for a long time [42-48]. To handle the problem of unknown dead-zone in control system design, an immediate method is to construct an adaptive dead-zone inverse [42]. Continuous and discrete adaptive dead-zone inverses were built for linear systems with unmeasurable dead-zone outputs $[43,44]$. Based on the assumption of the consistent dead-zone slopes in the positive and negative regions, a robust adaptive control approach was given for a class of special nonlinear systems without using the dead-zone inverse [45]. In [46, 47], the dead-zone is reconstructed into the form of a linear system with a static time-varying gain and bounded disturbances by introducing characteristic function. In [48], input dead-zone is taken into account and it is proved that the simplest ILC scheme retains its ability of achieving the satisfactory performance in tracking control. To the best of our knowledge, there is little work from the viewpoint of AILC to deal with nonlinear systems with time-delay and dead-zone nonlinearity in the literature at present stage.

In this paper, we present a novel AILC scheme for a class of nonlinear time-varying systems with unknown timevarying delays and unknown input dead-zone. To the best of our knowledge, up to now, few works have been reported in the field of AILC to deal with such kinds of systems. The main design difficulty comes from how to deal with dead-zone nonlinearity and delay-dependent uncertainty. In our work, the dead-zone output is represented as a novel simple nonlinear system with a time-varying gain, which is more general than the linear form in [36]. The approach removes the assumption of linear function outside the deadband without necessarily constructing a dead-zone inverse. An appropriate Lyapunov-Krasovskii functional and Young's inequality are combined to eliminate the unknown timevarying delays such that the design of the control law is free from these uncertainties. Furthermore, the possible singularity which may be caused by the appearance of the reciprocal of tracking error is avoided by employing the hyperbolic tangent function. By constructing a Lyapunovlike CEF, the stability conclusion is obtained in two cases by exploiting the properties of the hyperbolic tangent function via a rigorous analysis. In addition, the boundary layer function is introduced to remove the requirement for identical initial condition which is required for the majority of ILC schemes.

The rest of this paper is organized as follows. The problem formulation and preliminaries are given in Section 2. The AILC design is developed in Section 3. The CEF-based stability analysis is presented in Section 4. A simulation example is presented to verify the validity of the proposed scheme in Section 5, followed by conclusions in Section 6 .

\section{Problem Formulation and Preliminaries}

2.1. Problem Formulation. Consider a class of nonlinear timevarying systems with unknown time-varying time-delays and dead-zone running on a finite time interval $[0, T]$ repeatedly which is given by

$$
\begin{aligned}
\dot{x}_{i, k}(t) & =x_{i+1, k}(t), \quad i=1, \ldots, n-1, \\
\dot{x}_{n, k}(t) & =f\left(X_{k}(t), X_{\tau, k}(t), \theta(t)\right)+b(t) u_{k}(t)+d(t), \\
y_{k}(t) & =x_{1, k}(t), \quad u_{k}(t)=D\left(v_{k}(t)\right), \quad t \in[0, T], \\
x_{i, k}(t) & =\omega_{i}(t), \quad t \in\left[-\tau_{\max }, 0\right), i=1, \ldots, n,
\end{aligned}
$$

where $t$ is the time, $k \in N$ denotes the times of iteration, $N$ is the integer set and denotes the sets of iteration times, $y_{k}(t) \epsilon$ $R$ and $x_{i, k}(t) \in R, i=1, \ldots, n$ are the system output and states, respectively, $X_{k}(t) \triangleq\left[x_{1, k}(t), \ldots, x_{n, k}(t)\right]^{T}$ is the state vector, $\tau(t)$ is unknown time-varying delay of states and $x_{i, k}^{\tau} \triangleq x_{i, k}(t-$ $\tau(t)), i=2, \ldots, n$, and $X_{\tau, k}(t)=\left[x_{1, k}^{\tau}(t), \ldots, x_{n, k}^{\tau}(t)\right]^{T}, f(\cdot, \cdot, \cdot)$ are unknown smooth functions, and $b(t)$ is the unknown continuous time-varying gain of the system input. $\theta(t)$ is unknown continuous time-varying parameter vector; $d(t)$ is unknown bounded external disturbance. $\omega_{i}(t)$ denote the initial functions for delayed states, $i=1, \ldots, n$. Consider $v_{k}(t) \in R$ is the control input and the actuator nonlinearity $D\left(v_{k}(t)\right)$ is described as a dead-zone characteristic.

In this paper, a reference trajectory vector is given by $X_{d}(t)=\left[y_{d}(t), \dot{y}_{d}(t), \ldots, y_{d}^{(n-1)}(t)\right]^{T}$. The tracking error vector is $e_{k}(t)=\left[e_{1, k}, e_{2, k}, \ldots, e_{n, k}\right]^{T}=X_{k}(t)-X_{d}(t)$. The control objective of this paper is to design an adaptive iterative learning controller $u_{k}(t)$, such that the tracking error $e_{k}(t)$ converges to a small neighborhood of the origin as $k \rightarrow$ $\infty$; that is, $\lim _{k \rightarrow \infty}\left\|e_{k}(t)\right\| \leq \varepsilon_{e \infty}$, while all the signals in the closed-loop system remain bounded, where $\varepsilon_{e \infty}$ is a small positive error tolerance which will be given in the subsequent context and $\|\cdot\|$ denotes the Euclidian norm. Define the filtered tracking error as $e_{s k}(t)=\left[\begin{array}{ll}\Lambda^{T} & 1\end{array}\right] e_{k}(t)$, where $\Lambda=$ $\left[\lambda_{1}, \lambda_{2}, \ldots, \lambda_{n-1}\right]^{T}$ and $\lambda_{1}, \ldots, \lambda_{n-1}$ are the coefficients of Hurwitz polynomial $H(s)=s^{n-1}+\lambda_{n-1} s^{n-2}+\cdots+\lambda_{1}$.

To facilitate control system design, we make the following reasonable assumptions for the system functions, unknown time delays, and reference signals. 
Assumption 1. The unknown state time-varying delay $\tau(t)$ satisfies $0 \leq \tau(t) \leq \tau_{\max }, \dot{\tau}(t) \leq \kappa<1$, where $\tau_{\max }$ and $\kappa$ are unknown positive constants.

Assumption 2. The unknown smooth functions $f(\cdot, \cdot, \cdot)$ satisfy inequality

$$
\begin{aligned}
\mid f( & \left.X_{k}, X_{\tau, k}, \theta(t)\right)-f\left(X_{d}, X_{d, \tau}, \theta(t)\right) \mid \\
\leq & \left\|X_{k}-X_{d}\right\| h_{1}\left(X_{k}, X_{d}\right) \xi_{1}(\theta) \\
& +\left\|X_{\tau, k}-X_{d, \tau}\right\| h_{2}\left(X_{\tau, k}, X_{d, \tau}\right) \xi_{2}(\theta),
\end{aligned}
$$

where $X_{d, \tau} \triangleq X_{d}(t-\tau(t)), h_{1}(\cdot, \cdot)$ and $h_{2}(\cdot, \cdot)$ are known positive smooth functions, and $\xi_{1}(\theta)$ and $\xi_{2}(\theta)$ are unknown smooth functions of $\theta(t)$.

Assumption 3. The sign of $b(t)$ is known; without loss of generality, we always assume $b(t)>0$.

Assumption 4 (see [23]). The initial state errors $e_{i, k}(0)$ at each iteration are not necessarily zero small and fixed, but they are assumed to be bounded.

Assumption 5. The reference state trajectory $X_{d}(t)$ is continuous, bounded, and available.

Assumption 6. The unknown external $d(t)$ is bounded; that is, $|d(t)| \leq d_{\max }$ with an unknown constant $d_{\max }$.

Remark 7. Assumption 1 is common in the control problem of time-varying delay systems, which guarantees that the time delay terms can be eliminated by using Lyapunov-Krasovskii functional. Moreover, Assumption 1 is milder than that in [39-41] as it does not require the true value of $\tau_{\max }$ and $\kappa$.

Remark 8. As $b(t)$ is continuous on $[0, T]$, there exist constants $0<b_{\min } \leq b_{\max }$ such that $b_{\min } \leq b(t) \leq b_{\max }$. However, the control gain bounds $b_{\min }$ and $b_{\max }$ are only required for analytical purposes; their true values are not necessarily known in the sense that they are not used for controller design.

2.2. Dead-Zone Characteristic. The dead-zone characteristic can be described as

$$
\begin{aligned}
u_{k}(t) & =D\left(v_{k}(t)\right) \\
& = \begin{cases}m(t)\left(v_{k}(t)-b_{r}\right) & \text { for } v_{k}(t) \geq b_{r}, \\
0 & \text { for } b_{l}<v_{k}(t)<b_{r}, \\
m(t)\left(v_{k}(t)-b_{l}\right) & \text { for } v_{k}(t) \leq b_{l},\end{cases}
\end{aligned}
$$

where $b_{r} \geq 0$ and $b_{l} \leq 0$ are unknown constants, $m(t)>0$ is unknown time-varying slopes, and $v_{k}(t)$ is the input and $u_{k}(t)$ is the output of dead-zone. A graphical representation of the dead-zone in this paper is shown in Figure 1.

The dead-zone output $u_{k}(t)$ is not available for measurement. We make the following assumption on the dead-zone parameters.

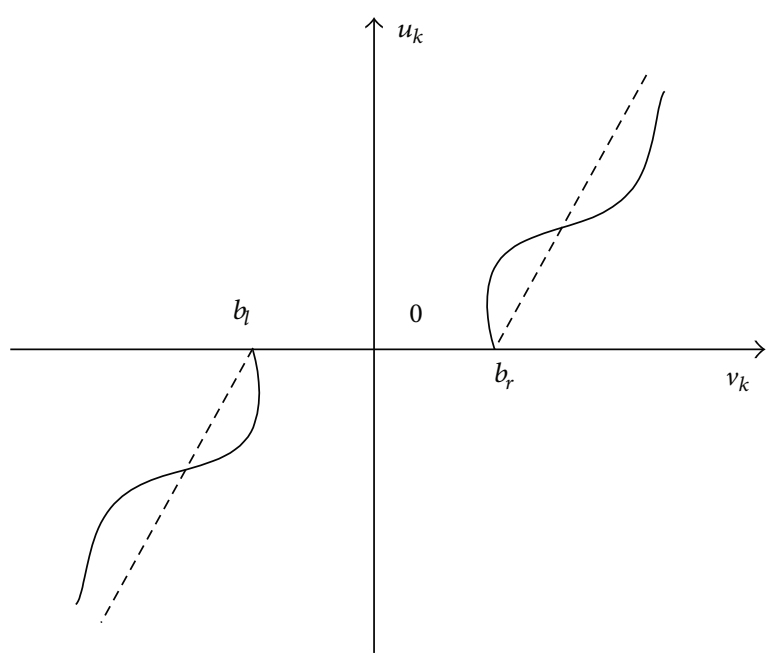

FIGURE 1: Dead-zone model.

Assumption 9. The dead-zone parameters $b_{r}, b_{l}$, and $m(t)$ are bounded. That is, there exist unknown constants $b_{r \text { min }}, b_{r \text { max }}$, $b_{l \text { min }}, b_{l \text { max }}, m_{\text {min }}$, and $m_{\max }$, such that $b_{r \text { min }} \leq b_{r} \leq b_{r \text { max }}$, $b_{l \min } \leq b_{l} \leq b_{l \max }$, and $m_{\min } \leq m(t) \leq m_{\max }$.

From a practical point of view, we can redefine the deadzone nonlinearity as

$$
u_{k}(t)=D\left(v_{k}\right)=m(t) v_{k}(t)-d_{1}\left(v_{k}(t)\right)
$$

with

$$
d_{1}\left(v_{k}(t)\right)= \begin{cases}m(t) b_{r} & \text { for } v_{k}(t) \geq b_{r} \\ m(t) v_{k}(t) & \text { for } b_{l}<v_{k}(t)<b_{r} \\ m(t) b_{l} & \text { for } v_{k}(t) \leq b_{l}\end{cases}
$$

It is obvious that $d_{1}\left(v_{k}(t)\right)$ is bounded.

Remark 10. Obviously, the dead-zone characteristic is nonlinear. And the form in [36] is the special case of (3) when $m(t)$ is invariant. Therefore, the presentation of dead-zone in our work is more general than the earlier results.

2.3. A Motivating Example. In order to clarify the main idea of AILC, we show the design procedure briefly by a simple scalar system running on $[0, T]$ as follows:

$$
\dot{z}_{k}(t)=\theta(t) \xi\left(z_{k}, t\right)+u_{k}^{z}(t),
$$

where $z_{k}(t)$ and $u_{k}^{z}(t)$ are the system state and the control input in the $k$ th iteration, respectively, $\theta(t)$ is an unknown time-varying parameter, and $\xi\left(z_{k}, t\right)$ is a known time-varying function. The reference trajectory is $z_{r}(t), t \in[0, T]$. Define the tracking error as $e_{k}^{z}(t)=z_{k}(t)-z_{r}(t)$ and design the control law and adaptive learning law for the unknown timevarying parameter for the $k$ th iteration as follows:

$$
\begin{aligned}
& u_{k}^{z}(t)=-k_{1} e_{k}^{z}(t)+\dot{z}_{r}(t)-\widehat{\theta}_{k}(t) \xi\left(z_{k}, t\right), \\
& \hat{\theta}_{k}(t)=\widehat{\theta}_{k-1}(t)+q \xi\left(z_{k}, t\right) e_{k}^{z}(t), \\
& \hat{\theta}_{0}(t)=0, \quad t \in[0, T],
\end{aligned}
$$


where $k_{1}, q>0$ are design parameters. Define the estimate error as $\widetilde{\theta}_{k}(t)=\widehat{\theta}_{k}(t)-\theta(t)$. Choose a Lyapunov-like CEF as

$$
E_{k}^{z}(t)=\frac{1}{2} e_{k}^{z}(t)+\frac{1}{2 q} \int_{0}^{t} \tilde{\theta}_{k}(\sigma) \mathrm{d} \sigma
$$

Throughout this paper, $\sigma$ denotes the integral variable. Then it can be derived that

$$
\Delta E_{k}^{z}(t)=E_{k}^{z}(t)-E_{k-1}^{z}(t) \leq-\int_{0}^{t}\left(e_{k}^{z}(\sigma)\right)^{2} \mathrm{~d} \sigma .
$$

We can further derive that

$$
\lim _{k \rightarrow \infty} \int_{0}^{T}\left(e_{k}^{z}(\sigma)\right)^{2} \mathrm{~d} \sigma=0
$$

Therefore, the system state $z_{k}(t)$ converges to the reference trajectory $z_{r}(t)$ on $[0, T]$ as $k \rightarrow \infty$.

\section{AILC Design}

According to Assumption 4, we know that there exist known constants $\varepsilon_{i}$ such that $\left|e_{i, k}(0)\right| \leq \varepsilon_{i}, i=1,2, \ldots n$ for any $k \in$ $N$. In order to relax the identical initial condition in ILC, we employ a boundary layer function [23] as follows:

$$
\begin{gathered}
s_{k}(t)=e_{s k}(t)-\eta(t) \operatorname{sat}\left(\frac{e_{s k}(t)}{\eta(t)}\right), \\
\eta(t)=\varepsilon e^{-K t}, \quad K>0,
\end{gathered}
$$

where $\varepsilon=\left[\begin{array}{ll}\Lambda^{T} & 1\end{array}\right]\left[\varepsilon_{1}, \varepsilon_{2}, \ldots, \varepsilon_{n}\right]^{T}$ and $K$ is a design parameter. The saturation function sat(.) is given by

$$
\operatorname{sat}\left(\frac{e_{s k}(t)}{\eta(t)}\right)= \begin{cases}1, & \text { if } e_{s k}(t)>\eta(t), \\ \frac{e_{s k}(t)}{\eta(t)}, & \text { if }\left|e_{s k}(t)\right| \leq \eta(t), \\ -1, & \text { if } e_{s k}(t)<-\eta(t)\end{cases}
$$

Remark 11. Note that $\eta(t)$ decreases along time axis with initial condition $\eta(0)=\varepsilon$ and $0<\eta(T) \leq \eta(t) \leq \varepsilon, \forall t \in[0, T]$, and then if $s_{k}(t)$ can be derived to zero $\forall t \in[0, T]$, the states will asymptotically converge to the reference trajectory for all $t \in[0, T]$.

It can be easily shown that

$$
\begin{aligned}
\left|e_{s k}(0)\right| & =\left|\lambda_{1} e_{1, k}(0)+\lambda_{2} e_{2, k}(0)+\cdots+e_{n, k}(0)\right| \\
& \leq \lambda_{1}\left|e_{1, k}(0)\right|+\lambda_{2}\left|e_{2, k}(0)\right|+\cdots+\left|e_{n, k}(0)\right| \\
& \leq \lambda_{1} \varepsilon_{1}+\lambda_{2} \varepsilon_{2}+\cdots+\varepsilon_{n}=\varepsilon=\eta(0)
\end{aligned}
$$

which implies that $s_{k}(0)=e_{s k}(0)-\eta(0)\left(e_{s k}(0) / \eta(0)\right)=0$ is satisfied forall $k \in N$. For the subsequent controller design, we firstly give the dynamic of $e_{n, k}(t)$ as follows:

$$
\begin{aligned}
\dot{e}_{n, k}(t)= & f\left(X_{k}(t), X_{\tau, k}, \theta(t)\right)+b(t) u_{k}+d(t)-y_{d}^{(n)}(t) \\
= & f\left(X_{k}, X_{\tau, k}, \theta(t)\right)-f\left(X_{d}, X_{d, \tau}, \theta(t)\right) \\
& +f\left(X_{d}, X_{d, \tau}, \theta(t)\right) \\
& +b(t)\left(m(t) v_{k}(t)-d_{1}\left(v_{k}(t)\right)\right)+d(t)-y_{d}^{(n)}(t) \\
= & f\left(X_{k}, X_{\tau, k}, \theta(t)\right)-f\left(X_{d}, X_{d, \tau}, \theta(t)\right) \\
& +f\left(X_{d}, X_{d, \tau}, \theta(t)\right)+b(t) m(t) v_{k}(t) \\
& +d_{2}(t)-y_{d}^{(n)}(t),
\end{aligned}
$$

where $d_{2}(t)=-b(t) d_{1}\left(v_{k}(t)\right)+d(t)$. By Assumptions 3 and 6 , we know that $d_{2}(t)$ is bounded; that is, there exists an unknown smooth positive function $\bar{d}(t)$ such that $\left|d_{2}(t)\right| \leq \bar{d}(t)$. For the simplicity of expression, we define $b_{m}(t)=b(t) m(t), \Theta(t)=f\left(X_{d}, X_{d, \tau}, \theta(t)\right)$, and $\Delta_{k}(t)$ $=f\left(X_{k}, X_{\tau, k}, \theta(t)\right)-f\left(X_{d}, X_{d, \tau}, \theta(t)\right)$. It is clear that $\Theta(t)$ is an unknown time-varying function which is invariant in the iteration domain and $\underline{b}_{m}=m_{\min } b_{\min } \leq b_{m}(t) \leq m_{\max } b_{\max }=$ $\bar{b}_{m}$. Define a smooth scalar function as

$$
V_{s_{k}}(t)=\frac{1}{2} s_{k}^{2}(t) .
$$

Differentiating $V_{s_{k}}(t)$ with respect to time, we can obtain

$$
\begin{aligned}
& \dot{V}_{s_{k}}(t)= s_{k}(t) \dot{s}_{k}(t) \\
&= \begin{cases}s_{k}(t)\left(\dot{e}_{s k}(t)-\dot{\eta}(t)\right), & \text { if } e_{s k}(t)>\eta(t) \\
0, & \text { if }\left|e_{s k}(t)\right| \leq \eta(t) \\
s_{k}(t)\left(\dot{e}_{s k}(t)+\dot{\eta}(t)\right), & \text { if } e_{s k}(t)<-\eta(t)\end{cases} \\
&= s_{k}(t)\left(\dot{e}_{s k}(t)-\dot{\eta}(t) \operatorname{sgn}\left(s_{k}(t)\right)\right) \\
&= s_{k}(t)\left(\begin{array}{l}
\sum_{j=1}^{n-1} \lambda_{j} e_{j+1, k}(t)-\dot{\eta}(t) \operatorname{sgn}\left(s_{k}(t)\right) \\
+\Theta(t)+\Delta_{k}(t)+b_{m}(t) v_{k}(t)
\end{array}\right. \\
&\left.\quad+d_{2}(t)-y_{d}^{(n)}(t)\right) \\
&=s_{k}(t)\left(\sum_{j=1}^{n-1} \lambda_{j} e_{j+1, k}(t)+K \eta(t) \operatorname{sgn}\left(s_{k}(t)\right)\right. \\
&+K e_{s k}(t)-K e_{s k}(t)+\Theta(t)+\Delta_{k}(t)
\end{aligned}
$$




$$
\begin{gathered}
\left.+b_{m}(t) v_{k}(t)+d_{2}(t)-y_{d}^{(n)}(t)\right) \\
=s_{k}(t)\left(\Theta(t)+\Delta_{k}(t)+b_{m}(t) v_{k}(t)\right. \\
\left.+\mu_{k}(t)+d_{2}(t)\right)-K s_{k}^{2}(t),
\end{gathered}
$$

where $\mu_{k}(t)=\sum_{j=1}^{n-1} \lambda_{j} e_{j+1, k}(t)+K e_{s k}(t)-y_{d}^{(n)}(t)$, and we use the relation

$$
\begin{gathered}
s_{k}(t)\left(-K e_{s k}(t)+K \eta(t) \operatorname{sgn}\left(s_{k}(t)\right)\right) \\
=s_{k}(t)\left(-K s_{k}(t)-K \eta(t) \operatorname{sat}\left(\frac{e_{s k}(t)}{\eta(t)}\right)\right. \\
\left.+K \eta(t) \operatorname{sgn}\left(s_{k}(t)\right)\right) \\
=-K s_{k}^{2}(t)-K \eta(t)\left|s_{k}(t)\right|+K \eta(t)\left|s_{k}(t)\right| \\
=-K s_{k}^{2}(t) .
\end{gathered}
$$

Utilizing Young's inequality and noting Assumption 2, it follows that

$$
\begin{aligned}
s_{k}(t) \Delta_{k}(t) \leq & \left|s_{k}(t)\right| \\
& \times\left(\left\|X_{k}-X_{d}\right\| h_{1}\left(X_{k}, X_{d}\right) \xi_{1}(\theta)\right. \\
& \left.+\left\|X_{\tau, k}-X_{d, \tau}\right\| h_{2}\left(X_{\tau, k}, X_{d, \tau}\right) \xi_{2}(\theta)\right) \\
\leq & \frac{1}{2} s_{k}^{2}(t) \xi_{1}^{2}(\theta)+\frac{1}{2}\left\|e_{k}\right\|^{2} h_{1}^{2}\left(X_{k}, X_{d}\right) \\
& +\frac{1}{2} s_{k}^{2}(t) \xi_{2}^{2}(\theta)+\frac{1}{2}\left\|e_{\tau, k}\right\|^{2} h_{2}^{2}\left(X_{\tau, k}, X_{d, \tau}\right) .
\end{aligned}
$$

Substituting (19) into (17) leads to

$$
\begin{aligned}
\dot{V}_{s_{k}}(t) \leq & s_{k}(t) \\
& \times\left(\Theta(t)+b_{m}(t) v_{k}(t)+\mu_{k}(t)+d_{2}(t)\right. \\
& \left.\quad+\frac{1}{2} s_{k}(t) \xi_{1}^{2}(\theta)+\frac{1}{2} s_{k}(t) \xi_{2}^{2}(\theta)\right)-K s_{k}^{2}(t) \\
& +\frac{1}{2}\left\|e_{k}\right\|^{2} h_{1}^{2}\left(X_{k}, X_{d}\right)+\frac{1}{2}\left\|e_{\tau, k}\right\|^{2} h_{2}^{2}\left(X_{\tau, k}, X_{d, \tau}\right) .
\end{aligned}
$$

To overcome the design difficulty arising from the unknown time-varying delay term, consider the following Lyapunov-Krasovskii functional:

$$
V_{U_{k}}(t)=\frac{1}{2(1-\kappa)} \int_{t-\tau(t)}^{t}\left\|e_{k}(\sigma)\right\|^{2} h_{2}^{2}\left(X_{k}(\sigma), X_{d}(\sigma)\right) \mathrm{d} \sigma
$$

Recalling Assumption 1, taking the time derivative of $V_{U_{k}}(t)$ leads to

$$
\begin{aligned}
\dot{V}_{U_{k}}(t)= & \frac{1}{2(1-\kappa)}\left\|e_{k}\right\|^{2} h_{2}^{2}\left(X_{k}, X_{d}\right) \\
& -\frac{1-\dot{\tau}(t)}{2(1-\kappa)}\left\|e_{\tau, k}\right\|^{2} h_{2}^{2}\left(X_{\tau, k}, X_{d, \tau}\right) \\
\leq & \frac{1}{2(1-\kappa)}\left\|e_{k}\right\|^{2} h_{2}^{2}\left(X_{k}, X_{d}\right) \\
& -\frac{1}{2}\left\|e_{\tau, k}\right\|^{2} h_{2}^{2}\left(X_{\tau, k}, X_{d, \tau}\right) .
\end{aligned}
$$

Define a Lyapunov functional as $V_{k}(t)=V_{s_{k}}(t)+V_{U_{k}}(t)$; combining (20) and (22), we can obtain

$$
\begin{aligned}
\dot{V}_{k}(t) \leq & s_{k}(t) \\
& \times\left(\Theta(t)+b_{m}(t) v_{k}(t)+\mu_{k}(t)+d_{2}(t)\right. \\
& \left.+\frac{1}{2} s_{k}(t) \xi_{1}^{2}(\theta)+\frac{1}{2} s_{k}(t) \xi_{2}^{2}(\theta)\right)-K s_{k}^{2}(t) \\
+ & \frac{1}{2}\left\|e_{k}\right\|^{2} h_{1}^{2}\left(X_{k}, X_{d}\right)+\frac{1}{2(1-\kappa)}\left\|e_{k}\right\|^{2} h_{2}^{2}\left(X_{k}, X_{d}\right) .
\end{aligned}
$$

For the convenience of expression, denote $\zeta_{k}(t)=(1 / 2)$ $\left\|e_{k}\right\|^{2} h_{1}^{2}\left(X_{k}, X_{d}\right)+(1 / 2(1-\kappa))\left\|e_{k}\right\|^{2} h_{2}^{2}\left(X_{k}, X_{d}\right)$ then (23) can be simplified as

$$
\dot{V}_{k}(t) \leq s_{k}(t)
$$

$$
\begin{aligned}
& \times\left(\Theta(t)+b_{m}(t) v_{k}(t)+\mu_{k}(t)+d_{2}(t)\right. \\
& \left.\quad+\frac{1}{2} s_{k}(t) \xi_{1}^{2}(\theta)+\frac{1}{2} s_{k}(t) \xi_{2}^{2}(\theta)+\frac{\zeta_{k}(t)}{s_{k}(t)}\right) \\
& -K s_{k}^{2}(t) .
\end{aligned}
$$

Here, we note that singularity problem may occur in (24) due to the term $\zeta_{k}(t) / s_{k}(t)$ which approaches $\infty$ as $s_{k}(t)$ approaches zero. In order to tackle this problem, we exploit the following characteristic of hyperbolic tangent function.

Lemma 12 (see [49]). For any constant $\eta>0$ and any variable $p \in R$

$$
\lim _{p \rightarrow 0} \frac{\tanh ^{2}(p / \eta)}{p}=0
$$


By employing the hyperbolic tangent function, (24) can be rewritten as

$$
\begin{aligned}
& \dot{V}_{k}(t) \leq s_{k}(t) \\
& \times\left(\Theta(t)+d_{2}(t)+b_{m}(t) v_{k}(t)+\mu_{k}(t)\right. \\
& \quad+\frac{1}{2} s_{k}(t) \xi_{1}^{2}(\theta)+\frac{1}{2} s_{k}(t) \xi_{2}^{2}(\theta) \\
&+\frac{a}{2 s_{k}(t)} \tanh ^{2}\left(\frac{s_{k}(t)}{\eta(t)}\right)\left\|e_{k}\right\|^{2} h_{1}^{2}\left(X_{k}, X_{d}\right) \\
&+\frac{a}{2(1-\kappa) s_{k}(t)} \tanh ^{2}\left(\frac{s_{k}(t)}{\eta(t)}\right) \\
&\left.\quad \times\left\|e_{k}\right\|^{2} h_{2}^{2}\left(X_{k}, X_{d}\right)\right) \\
&-K s_{k}^{2}(t)+\left(1-a \tanh ^{2}\left(\frac{s_{k}(t)}{\eta(t)}\right)\right) \zeta_{k}(t),
\end{aligned}
$$

where $a>1$ is a constant. From Lemma 12, we know that $\lim _{s_{k}(t) \rightarrow 0}\left(a / s_{k}(t)\right) \tanh ^{2}\left(s_{k}(t) / \eta(t)\right) \zeta_{k}(t)=0$. Hence, $\left(a / s_{k}(t)\right) \tanh ^{2}\left(s_{k}(t) / \eta(t)\right) \zeta_{k}(t)$ is defined at $s_{k}(t)=0$ and the possible singularity problem has been avoided. Upon multiplication of $(26)$ by $1 / b_{m}(t)$, it becomes

$$
\begin{aligned}
& \frac{\dot{V}_{k}(t)}{b_{m}(t)} \leq s_{k}(t) \\
& \times\left(\frac{1}{b_{m}(t)}\left(\Theta(t)+d_{2}(t)\right)+v_{k}(t)+\frac{1}{b_{m}(t)} \mu_{k}(t)\right. \\
& +\frac{1}{2 b_{m}(t)} s_{k}(t)\left(\xi_{1}^{2}(\theta)+\xi_{2}^{2}(\theta)\right) \\
& +\frac{a}{2 b_{m}(t) s_{k}(t)} \tanh ^{2}\left(\frac{s_{k}(t)}{\eta(t)}\right) \\
& \times\left\|e_{k}\right\|^{2} h_{1}^{2}\left(X_{k}, X_{d}\right) \\
& +\frac{a}{2 b_{m}(t)(1-\kappa) s_{k}(t)} \tanh ^{2}\left(\frac{s_{k}(t)}{\eta(t)}\right) \\
& \left.\times\left\|e_{k}\right\|^{2} h_{2}^{2}\left(X_{k}, X_{d}\right)\right) \\
& -\frac{K}{b_{m}(t)} s_{k}^{2}(t)+\frac{1}{b_{m}(t)} \\
& \times\left(1-a \tanh ^{2}\left(\frac{s_{k}(t)}{\eta(t)}\right)\right) \zeta_{k}(t) \\
& =s_{k}(t)\left(\beta^{T}(t) \Phi_{k}(t)+v_{k}(t)\right)-\frac{K}{b_{m}(t)} s_{k}^{2}(t) \\
& +\frac{1}{b_{m}(t)}\left(1-a \tanh ^{2}\left(\frac{s_{k}(t)}{\eta(t)}\right)\right) \zeta_{k}(t),
\end{aligned}
$$

where $\beta(t)=\left[\left(1 / b_{m}(t)\right)\left(\Theta(t)+d_{2}(t)\right), 1 / b_{m}(t),\left(1 / b_{m}(t)\right)\right.$ $\left.\left(\xi_{1}^{2}(\theta)+\xi_{2}^{2}(\theta)\right),\left(1 /(1-\kappa) b_{m}(t)\right)\right]^{T}$ denote the unknown time-varying parameter vector that is invariant along the iteration axis and $\Phi_{k}(t)=\left[1, \mu_{k}(t)+\left(a / 2 s_{k}(t)\right)\right.$ $\tanh ^{2}\left(s_{k}(t) / \eta(t)\right)\left\|e_{k}\right\|^{2} h_{1}^{2}\left(X_{k}, X_{d}\right), s_{k}(t), \quad\left(a / 2 s_{k}(t)\right) \tanh ^{2}\left(s_{k}\right.$ $\left.(t) / \eta(t))\left\|e_{k}\right\|^{2} h_{2}^{2}\left(X_{k}, X_{d}\right)\right]^{T}$. Based on (27), we can design the adaptive iterative learning controller as follows:

$$
v_{k}(t)=-\widehat{\beta}_{k}(t) \Phi_{k}(t)-K_{1} s_{k}(t),
$$

where $K_{1}>0$ is design parameters and $\widehat{\beta}_{k}(t)$ is the estimate of $\beta(t)$ in the $k$ th iteration. The adaptive learning algorithms for unknown parameter are given by

$$
\begin{gathered}
\widehat{\beta}_{k}(t)=\widehat{\beta}_{k-1}(t)+q s_{k}(t) \Phi_{k}(t), \\
\widehat{\beta}_{0}(t)=0, \quad t \in[0, T],
\end{gathered}
$$

where $q>0$ is the learning gain. Define the estimation error as $\widetilde{\beta}_{k}(t)=\widehat{\beta}_{k}(t)-\beta(t)$. Hence, substituting the controller (28) back into (27) yields

$$
\begin{aligned}
\frac{\dot{V}_{k}(t)}{b_{m}(t)} \leq & -s_{k}(t) \widetilde{\beta}_{k}^{T}(t) \Phi_{k}(t)-\left(\frac{K}{b_{m}(t)}+K_{1}\right) s_{k}^{2}(t) \\
& +\frac{1}{b_{m}(t)}\left(1-a \tanh ^{2}\left(\frac{s_{k}(t)}{\eta(t)}\right)\right) \zeta_{k}(t) \\
\leq & -s_{k}(t) \widetilde{\beta}_{k}^{T}(t) \Phi_{k}(t)-\left(\frac{K}{\bar{b}_{m}}+K_{1}\right) s_{k}^{2}(t) \\
& +\frac{1}{b_{m}(t)}\left(1-a \tanh ^{2}\left(\frac{s_{k}(t)}{\eta(t)}\right)\right) \zeta_{k}(t) .
\end{aligned}
$$

For simplicity in expression, we denote $\left(K / \bar{b}_{m}+K_{1}\right)$ by $K_{2}=\left(K / \bar{b}_{m}+K_{1}\right)$. Then, (30) can be continued as

$$
\begin{aligned}
s_{k}(t) \widetilde{\beta}_{k}^{T}(t) \Phi_{k}(t) \leq & -\frac{\dot{V}_{k}(t)}{b_{m}(t)}-K_{2} s_{k}^{2}(t) \\
& +\frac{1}{b_{m}(t)}\left(1-a \tanh ^{2}\left(\frac{s_{k}(t)}{\eta(t)}\right)\right) \zeta_{k}(t) .
\end{aligned}
$$

\section{Stability and Convergence Analysis}

In this section, we will check the stability of the closed-loop system and the convergence of tracking errors by CEF-based analysis. First of all, we give the following property of the tangent hyperbolic function.

Lemma 13. Define a compact set $\Omega_{s_{k}}$ as $\Omega_{s_{k}}:=\left\{s_{k}(t) \mid\right.$ $\left.\left|s_{k}(t)\right| \leq m \eta(t)\right\}$. Then, for any $s_{k}(t) \notin \Omega_{s_{k}}$, the following inequality holds:

$$
1-a \tanh ^{2}\left(\frac{s_{k}(t)}{\eta(t)}\right)<0
$$

where $m=\ln (\sqrt{a /(a-1)}+\sqrt{1 /(a-1)})$. 
Proof. See the appendix.

The stability and convergence property of the proposed AILC scheme is summarized as follows.

Theorem 14. Considering closed-loop system (1), if Assumptions 1-6 and 9 hold, designing the control laws (28) with adaptive updating laws (29), the following properties can be guaranteed: (i) all the signals of the closed-loop system are bounded; (ii) the filtered tracking error $e_{s k}(t)$ converges to a small neighborhood of zero as $k \rightarrow \infty$ in $L_{2}$ norm; that is, $\lim _{k \rightarrow \infty} \int_{0}^{T}\left(e_{s k}(\sigma)\right)^{2} \mathrm{~d} \sigma \leq \varepsilon_{e s k}, \varepsilon_{e s k}=(1 / 2 K)(1+m)^{2} \varepsilon^{2} ;$ and (iii) the tracking error vector satisfies $\lim _{k \rightarrow \infty}\left\|e_{k}(t)\right\| \leq \varepsilon_{e \infty}$, $\varepsilon_{e \infty}=(1+\|\Lambda\|)\left(k_{0} \sum_{i=1}^{n-1} \varepsilon_{i}+\left(1 /\left(\lambda_{0}-K\right)\right)(1+m) \varepsilon k_{0}\right)+(1+m) \varepsilon$, where $\lambda_{0}$ and $k_{0}$ are positive constants and will be given later.

Proof. Define a Lyapunov-like CEF as follows:

$$
E_{k}(t)=\frac{1}{2 q} \int_{0}^{t} \widetilde{\beta}_{k}^{T}(\sigma) \widetilde{\beta}_{k}(\sigma) \mathrm{d} \sigma .
$$

The difference of $E_{k}(t)$ is

$$
\begin{aligned}
\Delta E_{k}(t) & =E_{k}(t)-E_{k-1}(t) \\
& =\frac{1}{2 q} \int_{0}^{t}\left(\widetilde{\beta}_{k}^{T}(\sigma) \widetilde{\beta}_{k}(\sigma)-\widetilde{\beta}_{k-1}^{T}(\sigma) \widetilde{\beta}_{k-1}(\sigma)\right) \mathrm{d} \sigma .
\end{aligned}
$$

Utilizing the algebraic relation $(a-b)^{T}(a-b)-(a-c)^{T}(a-$ $c)=(c-b)^{T}[2(a-b)+(b-c)]$ and taking adaptive learning law (29) into account, we have the following inequality:

$$
\begin{aligned}
\Delta E_{k}(t)= & \int_{0}^{t} s_{k}(\sigma) \widetilde{\beta}_{k}^{T}(\sigma) \Phi_{k}(\sigma) \mathrm{d} \sigma \\
& -\frac{q}{2} \int_{0}^{t} s_{k}^{2}(\sigma)\left\|\Phi_{k}(\sigma)\right\|^{2} \mathrm{~d} \sigma \\
\leq & \int_{0}^{t} s_{k}(\sigma) \widetilde{\beta}_{k}^{T}(\sigma) \Phi_{k}(\sigma) \mathrm{d} \sigma .
\end{aligned}
$$

Substituting (31) into (35), it follows that

$$
\begin{aligned}
\Delta E_{k}(t) \leq & -\int_{0}^{t} \frac{\dot{V}_{k}(\sigma)}{b_{m}(\sigma)} \mathrm{d} \sigma \\
& +\int_{0}^{t} \frac{1}{b_{m}(\sigma)}\left(1-a \tanh ^{2}\left(\frac{s_{k}(\sigma)}{\eta(\sigma)}\right)\right) \zeta_{k}(\sigma) \mathrm{d} \sigma \\
& -\int_{0}^{t} K_{2} s_{k}^{2}(\sigma) \mathrm{d} \sigma \\
\leq & -\frac{1}{\bar{b}_{m}} V_{k}(t)-K_{2} \int_{0}^{t} s_{k}^{2}(\sigma) \mathrm{d} \sigma \\
& +\int_{0}^{t} \frac{1}{b_{m}(\sigma)}\left(1-a \tanh ^{2}\left(\frac{s_{k}(\sigma)}{\eta(\sigma)}\right)\right) \zeta_{k}(\sigma) \mathrm{d} \sigma .
\end{aligned}
$$

For analysis of stability, we consider two cases.
Case 1. Consider $\left(s_{k}(t) \in \Omega_{s_{k}}\right)$. When $s_{k}(t) \in \Omega_{s_{k}},\left|s_{k}(t)\right| \leq$ $m \eta(t)$ holds. If $s_{k}(t)=0$, we know $e_{s k}(t)$ is bounded by $\eta(t)$; that is, $\left|e_{s k}(t)\right| \leq \eta(t)$. If $s_{k}(t)>0$, we have $s_{k}(t)=e_{s k}(t)-\eta(t)$, and from $\left|s_{k}(t)\right| \leq m \eta(t)$ we can obtain $s_{k}(t)=e_{s k}(t)-\eta(t) \leq$ $m \eta(t)$ which further implies $0<e_{s k} \leq(1+m) \eta(t)$. Similarly, if $s_{k}(t)<0$, we have $s_{k}(t)=e_{s k}(t)+\eta(t) \geq-m \eta(t)$ which means $0>e_{s k}(t) \geq-(1+m) \eta(t)$. Synthesizing the above analysis, we know that $\left|e_{s k}(t)\right| \leq(1+m) \eta(t)$ holds. Obviously, $x_{i, k}(t)$ are bounded since $X_{d}(t)$ is bounded. According to the smoothness of $h_{1}(\cdot, \cdot)$ and $h_{2}(\cdot, \cdot)$, we know that $\Phi_{k}(t)$ is a bounded vector. Recalling updating law (29), we know $\widehat{\beta}_{0}(t)=0, t \in[0, T]$; then, when $s_{k}(t) \in \Omega_{s_{k}}, \widehat{\beta}_{k}(t)$ is bounded as well, $k \in N$. Following this chain of reasoning, the boundedness of $v_{k}(t)$ can be deduced. As such, all closedloop signals are bounded.

Remark 15 . Theoretically, $m$ can be made arbitrarily small by choosing $a$, for example, when we choose $a=100$ and $m=0.099$. This leads to $s_{k}$ arbitrarily small. However, large a may give rise to high gain control which can deteriorate the transient performance of closed-loop system. Consequently, in practical applications, the designers should choose appropriate design parameters to gain satisfactory transient performance and the ideal tracking error.

Case 2. Consider $\left(s_{k}(t) \notin \Omega_{s_{k}}\right)$. According to Lemma 13, we know that the last term of $\Delta E_{k}(t)$ can be removed from the analysis. Therefore, (36) can be simplified as

$$
\Delta E_{k}(t) \leq-\frac{1}{\bar{b}_{m}} V_{k}(t)-K_{2} \int_{0}^{t} s_{k}^{2}(\sigma) \mathrm{d} \sigma<0 .
$$

Inequality (37) shows that $E_{k}(t)$ is decreasing along iteration axis. Thus, the boundedness of $E_{k}(t)$ can be guaranteed as long as $E_{1}(t)$ is finite. According to the definition $E_{k}, E_{1}(t)$ is given by

$$
E_{1}(t)=\frac{1}{2 q} \int_{0}^{t} \widetilde{\beta}_{1}^{T}(\sigma) \widetilde{\beta}_{1}(\sigma) \mathrm{d} \sigma
$$

Taking the time derivative of $E_{1}(t)$ results in

$$
\dot{E}_{1}(t)=\frac{1}{2 q} \widetilde{\beta}_{1}^{T}(t) \widetilde{\beta}_{1}(t) .
$$

Recalling parameter adaptive laws (29), we have $\widehat{\beta}_{1}(t)=$ $q s_{1}(t) \Phi_{1}(t)$, and then we obtain

$$
\begin{aligned}
\dot{E}_{1}(t) & =\frac{1}{2 q} \widetilde{\beta}_{1}^{T}(t) \widetilde{\beta}_{1}(t) \\
& =\frac{1}{2 q}\left(\widetilde{\beta}_{1}^{T}(t) \widetilde{\beta}_{1}(t)-2 \widetilde{\beta}_{1}^{T}(t) \widehat{\beta}_{1}(t)\right)+\frac{1}{q} \widetilde{\beta}_{1}^{T}(t) \widehat{\beta}_{1}(t) \\
& =\frac{1}{2 q}\left(\left(\widehat{\beta}_{1}(t)-\beta(t)\right)^{T}\left(\widehat{\beta}_{1}(t)-\beta(t)\right)\right.
\end{aligned}
$$




$$
\begin{gathered}
\left.-2\left(\widehat{\beta}_{1}(t)-\beta(t)\right)^{T} \widehat{\beta}_{1}(t)\right) \\
+s_{1}(t) \widetilde{\beta}_{1}^{T}(t) \Phi_{1}(t) \\
=\frac{1}{2 q}\left(-\widehat{\beta}_{1}^{T}(t) \widehat{\beta}_{1}+\beta^{T}(t) \beta(t)\right)+s_{1}(t) \widetilde{\beta}_{1}^{T}(t) \Phi_{1}(t) .
\end{gathered}
$$

Substituting (31) into (40) yields

$$
\dot{E}_{1}(t) \leq-\frac{\dot{V}_{1}(t)}{b_{m}(t)}-K_{2} s_{1}^{2}(t)+\frac{1}{2 q} \beta^{T}(t) \beta(t) .
$$

Denote $\beta_{\max }=\max _{t \in[0, T]}\left\{(1 / 2 q) \beta^{T}(t) \beta(t)\right\}$. Integrating the above inequality over $[0, t]$ leads to

$$
E_{1}(t)-E_{1}(0) \leq-\frac{1}{\bar{b}_{m}} V_{1}(t)-K_{2} \int_{0}^{t} s_{1}^{2}(\sigma) \mathrm{d} \sigma+t \cdot \beta_{\max } .
$$

Obviously, $E_{1}(0)=0$, and then it follows from (42) that

$$
E_{1}(t) \leq t \cdot \beta_{\max }<\infty
$$

which indicates the boundedness of $E_{1}(t)$, so $E_{k}(t)$ is finite for any $k \in N$. Using (37) repeatedly, we have

$$
\begin{aligned}
E_{k}(t) & =E_{1}(t)+\sum_{l=2}^{k} \Delta E_{l}(t) \\
& <E_{1}(t)-\frac{1}{\bar{b}_{m}} \sum_{l=2}^{k} V_{l}(t)-\sum_{l=2}^{k} K_{2} \int_{0}^{t} s_{l}^{2}(\sigma) \mathrm{d} \sigma \\
& \leq E_{1}(t)-\sum_{l=2}^{k} K_{2} \int_{0}^{t} s_{l}^{2}(\sigma) \mathrm{d} \sigma .
\end{aligned}
$$

We rewrite inequality (44) as

$$
\sum_{l=2}^{k} K_{2} \int_{0}^{t} s_{l}^{2}(\sigma) \mathrm{d} \sigma \leq\left(E_{1}(t)-E_{k}(t)\right) \leq E_{1}(t) .
$$

Let $t=T$, and, taking the limitation of (45), it follows that

$$
\lim _{k \rightarrow \infty} \sum_{l=2}^{k} \int_{0}^{T} s_{l}^{2}(\sigma) \mathrm{d} \sigma \leq \frac{1}{K_{2}} E_{1}(T) .
$$

Since $E_{1}(T)$ is bounded, with the aid of the convergence theorem of the sum of series, $\lim _{k \rightarrow \infty} \int_{0}^{T} s_{k}^{2}(\sigma) \mathrm{d} \sigma=0$, which implies that $\lim _{k \rightarrow \infty} s_{k}(t)=s_{\infty}(t)=0, \forall t \in[0, T]$. Moreover, from definition (11), we can know that, when $\left|e_{s k}(t)\right| \leq$ $\eta(t), s_{k}(t)=0$, then $\lim _{k \rightarrow \infty} \int_{0}^{T} s_{k}^{2}(\sigma) \mathrm{d} \sigma=0$ is equivalent to $\lim _{k \rightarrow \infty}\left|e_{s k}(t)\right| \leq \eta(t)$, which furthermore implies that $\lim _{k \rightarrow \infty} \int_{0}^{T}\left(e_{s k}(\sigma)\right)^{2} \mathrm{~d} \sigma \leq \int_{0}^{T}(\eta(\sigma))^{2} \mathrm{~d} \sigma$.

According to the boundedness of $E_{k}(t)$, we can obtain the boundedness of $\widehat{\beta}_{k}(t)$. From $\int_{0}^{t} s_{k}^{2}(\sigma) \mathrm{d} \sigma \leq \int_{0}^{T} s_{k}^{2}(\sigma) \mathrm{d} \sigma$, we can get the boundedness of $s_{k}(t)$. Considering the finiteness of reference trajectory $X_{d}(t)$, we further obtain that $x_{i, k}(t)$ are bounded. Based on the above reasoning, we can obtain the boundedness of $v_{k}(t)$ by similar analysis in Case 1 .

Synthesizing the derivations in two cases, we can conclude that the proposed control algorithm is able to guarantee that all closed-loop signals are bounded and $\lim _{k \rightarrow \infty}\left|e_{s k}(t)\right| \leq(1+m) \eta(t)$. Therefore, we can obtain $\lim _{k \rightarrow \infty} \int_{0}^{T}\left(e_{s k}(\sigma)\right)^{2} \mathrm{~d} \sigma \leq \varepsilon_{e}, \varepsilon_{e}=\int_{0}^{T}((1+m) \eta(\sigma))^{2} \mathrm{~d} \sigma=$ $(1 / 2 K)(1+m)^{2} \varepsilon^{2}\left(1-e^{-2 K T}\right) \leq(1 / 2 K)(1+m)^{2} \varepsilon^{2}=\varepsilon_{e s k}$; thus, the control objective is achieved. Furthermore, the bound of $e_{s \infty}(t)$ will satisfy $\lim _{k \rightarrow \infty}\left|e_{s k}(t)\right|=e_{s \infty}(t)=(1+m) \varepsilon e^{-K t}$, $\forall t \in[0, T]$.

Define the vector $\psi_{k}(t)=\left[e_{1, k}(t), e_{2, k}(t), \ldots, e_{n-1, k}(t)\right]^{T}$, and then a state representation of $e_{s k}(t)=\left[\begin{array}{ll}\Lambda^{T} & 1\end{array}\right] e_{k}(t)$ can be expressed as

$$
\dot{\psi}_{k}(t)=A_{s} \psi_{k}(t)+b_{s} e_{s k}(t)
$$

where

$$
\begin{gathered}
A_{s}=\left[\begin{array}{cccc}
0 & 1 & \cdots & 0 \\
\vdots & \vdots & \ddots & \vdots \\
0 & 0 & \cdots & 1 \\
-\lambda_{1} & -\lambda_{2} & \cdots & -\lambda_{n-1}
\end{array}\right] \in R^{(n-1) \times(n-1)} \\
b_{s}=\left[\begin{array}{c}
0 \\
\vdots \\
0 \\
1
\end{array}\right] \in R^{n-1}
\end{gathered}
$$

with $A_{s}$ as a stable matrix. In addition, there are two constants $k_{0}>0$ and $\lambda_{0}>0$ such that $\left\|e^{A_{s} t}\right\| \leq k_{0} e^{-\lambda_{0} t}$ [50]. The solution for $\dot{\psi}_{k}(t)$ is

$$
\psi_{k}(t)=e^{A_{s} t} \psi_{k}(0)+\int_{0}^{t} e^{A_{s}(t-\sigma)} b_{s}\left|e_{s k}(\sigma)\right| \mathrm{d} \sigma
$$

Accordingly, it follows from (49) that

$$
\left\|\psi_{k}(t)\right\|=k_{0}\left\|\psi_{k}(0)\right\| e^{-\lambda_{0} t}+k_{0} \int_{0}^{t} e^{-\lambda_{0}(t-\sigma)}\left|e_{s k}(\sigma)\right| \mathrm{d} \sigma .
$$

When we choose suitable parameters such that $\lambda_{0}>K$, from $\lim _{k \rightarrow \infty}\left|e_{s k}(t)\right| \leq(1+m) \eta(t)$, we can have

$$
\begin{aligned}
\left\|\psi_{\infty}(t)\right\| & =k_{0}\left\|\psi_{\infty}(0)\right\| e^{-\lambda_{0} t}+k_{0} \int_{0}^{t} e^{-\lambda_{0}(t-\sigma)}\left|e_{s \infty}(\sigma)\right| \mathrm{d} \sigma \\
& \leq k_{0}\left\|\psi_{\infty}(0)\right\|+(1+m) \varepsilon k_{0} \int_{0}^{t} e^{-\lambda_{0}(t-\sigma)} e^{-K \sigma} \mathrm{d} \sigma \\
& =k_{0}\left\|\psi_{\infty}(0)\right\|+(1+m) \varepsilon k_{0} \frac{1}{\lambda_{0}-K}\left(e^{-K t}-e^{-\lambda_{0} t}\right) \\
& \leq k_{0}\left\|\psi_{\infty}(0)\right\|+\frac{1}{\lambda_{0}-K}(1+m) \varepsilon k_{0} .
\end{aligned}
$$


Noting $e_{s k}(t)=\left[\begin{array}{ll}\Lambda^{T} & 1\end{array}\right] e_{k}(t)$ and $e_{k}(t)=\left[\begin{array}{ll}\psi_{k}^{T}(t) & e_{n, k}(t)\end{array}\right]^{T}$, we have

$$
\begin{aligned}
\left\|e_{k}(t)\right\| & \leq\left\|\psi_{k}(t)\right\|+\left|e_{n, k}(t)\right| \\
& =\left\|\psi_{k}(t)\right\|+\left|e_{s k}(t)-\Lambda^{T} \psi_{k}(t)\right| \\
& \leq(1+\|\Lambda\|)\left\|\psi_{k}(t)\right\|+\left|e_{s k}(t)\right| .
\end{aligned}
$$

Combining the previous two inequalities, we can obtain

$$
\begin{aligned}
\left\|e_{\infty}(t)\right\| \leq & (1+\|\Lambda\|)\left\|\psi_{\infty}(t)\right\|+\left|e_{s \infty}(t)\right| \\
\leq & (1+\|\Lambda\|)\left(k_{0}\left\|\psi_{\infty}(0)\right\|+\frac{1}{\lambda_{0}-K}(1+m) \varepsilon k_{0}\right) \\
& +(1+m) \eta(t) \\
\leq & (1+\|\Lambda\|)\left(k_{0} \sum_{i=1}^{n-1} \varepsilon_{i}+\frac{1}{\lambda_{0}-K}(1+m) \varepsilon k_{0}\right) \\
& +(1+m) \eta(t) \\
\leq & (1+\|\Lambda\|)\left(k_{0} \sum_{i=1}^{n-1} \varepsilon_{i}+\frac{1}{\lambda_{0}-K}(1+m) \varepsilon k_{0}\right) \\
& +(1+m) \varepsilon=\varepsilon_{e \infty} .
\end{aligned}
$$

This concludes the proof.

\section{Simulation Studies}

In this section, a simulation example is presented to verify the effectiveness of the AILC scheme. Consider the following second-order nonlinear system with unknown time-varying delays and unknown dead-zone running on $[0,10]$, repetitively:

$$
\begin{gathered}
\dot{x}_{1, k}(t)=x_{2, k}(t), \\
\dot{x}_{2, k}(t)=f\left(X_{k}(t), X_{\tau, k}(t), \theta(t)\right)+b(t) u_{k}(t)+d(t), \\
y_{k}(t)=x_{1, k}(t), \quad u_{k}(t)=D\left(v_{k}(t)\right),
\end{gathered}
$$

where $f\left(X_{k}(t), X_{\tau, k}(t), \theta(t)\right)=-\left(x_{1, k}(t)+x_{2, k}(t)\right) \theta(t)+$ $\exp \left(-\theta(t)\left(\left(x_{1, k}^{\tau}(t)\right)^{2}+\left(x_{2, k}^{\tau}(t)\right)^{2}\right)\right), b(t)=2+0.5 \sin t, d(t)=$ $0.1 \sin t$, and the time delays are $\tau(t)=0.5(1+\sin t)$ with $\tau_{\max }=1, \theta(t)=|\cos (t)|$. It can be easily verified that

$$
\begin{aligned}
& \left|\exp \left(-\theta(t)\left\|X_{k}\right\|^{2}\right)-\exp \left(-\theta(t)\left\|X_{d}\right\|^{2}\right)\right| \\
& \leq\left\|X_{k}-X_{d}\right\| \sqrt{2|\theta(t)| e^{-0.5}} .
\end{aligned}
$$

Obviously, Assumptions 1-3 and Assumptions 5, 6, and 9 are satisfied. Moreover, we can know that $h_{1}=1$ and $h_{2}=1$. We give the simulation study in the following three cases.

Case 1. The reference trajectory to be tracked by the state vector is given by $X_{d}(t)=[\sin t, \cos t]^{T}$. The design

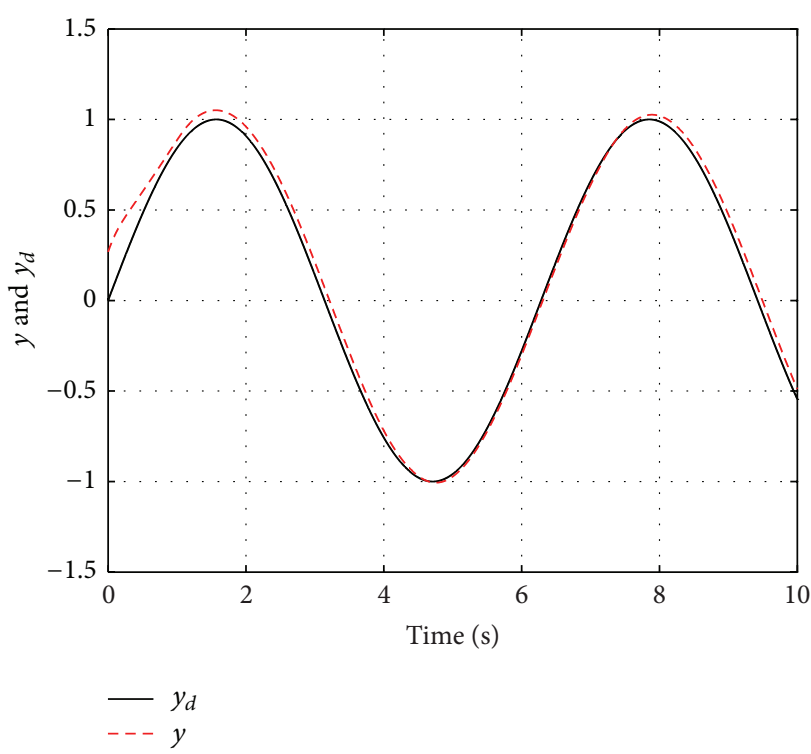

FIGURE 2: System output $y$ on $y_{d}(k=1)$ in Case 1 .

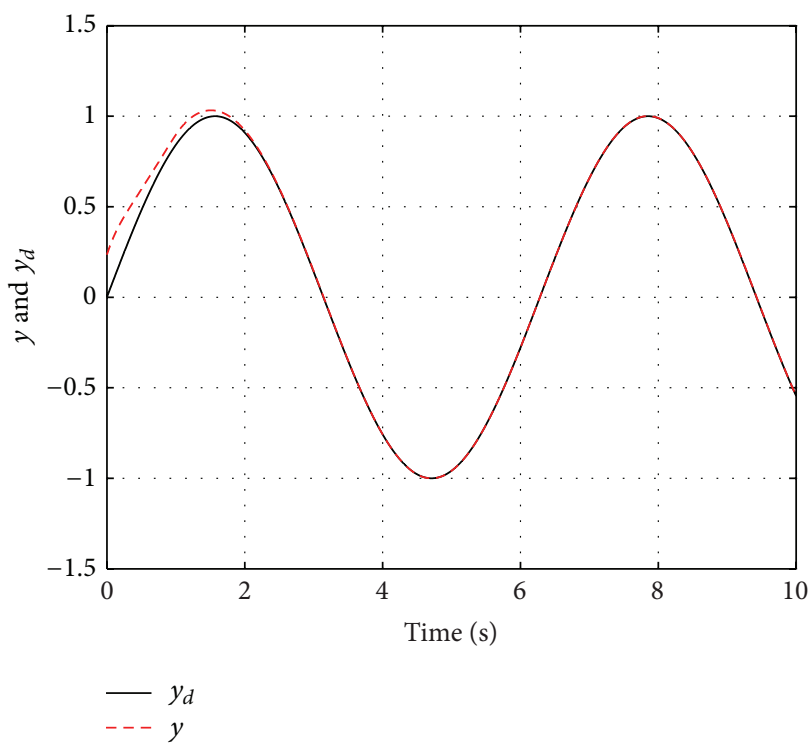

Figure 3: System output $y$ on $y_{d}(k=10)$ in Case 1.

parameters are chosen as $\varepsilon_{1}=\varepsilon_{2}=1, \lambda=2, K=3, \gamma=0.5$, $K_{1}=2, q=1, a=5, \varepsilon=\lambda \varepsilon_{1}+\varepsilon_{2}=3$. The parameters for dead-zone are specified by $m=1+0.2 \sin t, b_{r}=0.25, b_{l}=$ -0.25 . The initial conditions $x_{1, k}(0)$ and $x_{2, k}(0)$ are randomly taken in the intervals $[-0.5,0.5]$ and $[0.5,1.5]$, respectively. Parts of the simulation results are shown in Figures 2, 3, 4, 5 , and 6 . From the simulation results, we can see that the proposed AILC is effective in the sense that it can drive the tracking errors converge to zero along the iteration axis.

Case 2. To show the control performance for more complicated reference trajectory, we choose the reference trajectory as $X_{d}(t)=[\sin t+\sin (1.5 t), \cos t+1.5 \cos (1.5 t)]^{T}$. The design parameters are chosen the same as those in case 1 . 


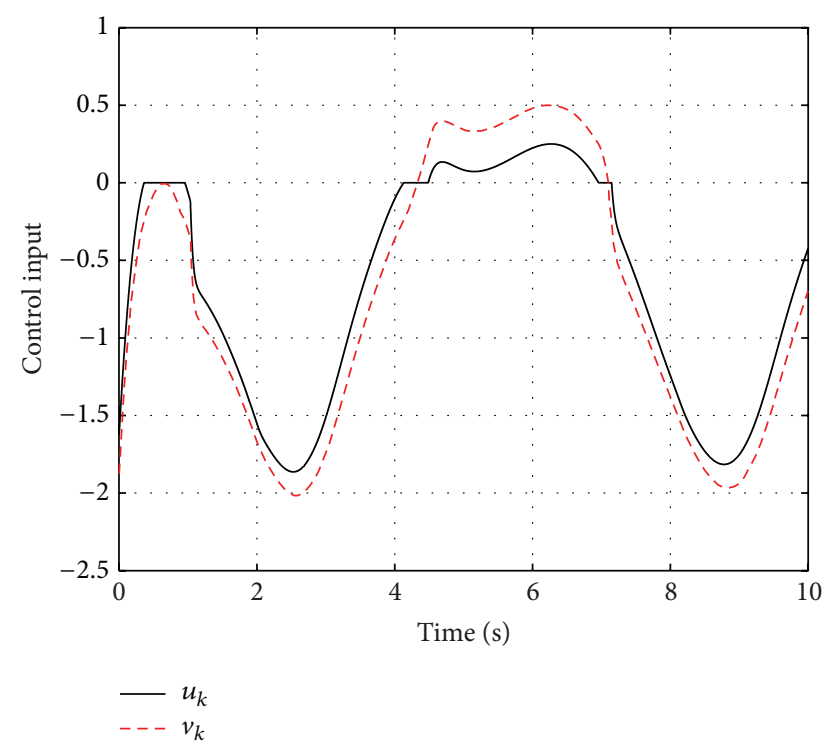

FIgURE 4: Control input $u_{k}$ and $v_{k}(k=1)$ in Case 1.

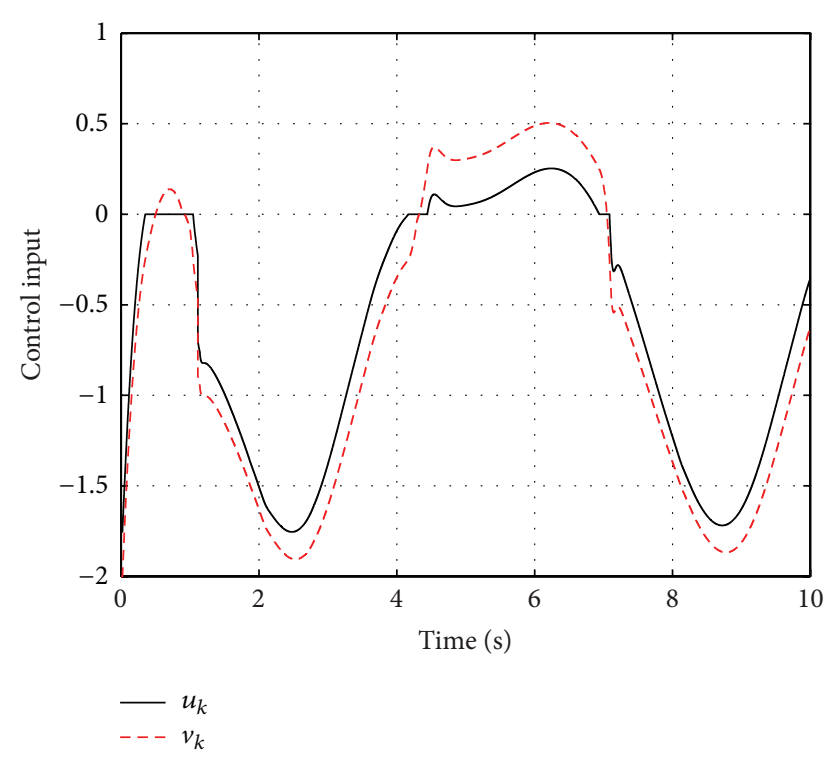

FIgure 5: Control input $u_{k}$ and $v_{k}(k=10)$ in Case 1.

The initial conditions $x_{1, k}(0)$ and $x_{2, k}(0)$ are randomly taken in the intervals $[-0.5,0.5]$ and $[2,3]$, respectively. Parts of the simulation results are shown in Figures 7, 8, 9, 10, and 11. It shows that for more complicated reference trajectory the proposed approach is also able to achieve excellent tracking performance.

Case 3. Finally, the contribution of this paper is shown by comparing the proposed controller with traditional adaptive controller. The controller is the same, but the adaptive laws using $\sigma$-modification for parameters are given by

$$
\dot{\hat{\beta}}(t)=-\Gamma\left[\Phi_{k}(t) s_{k}+\sigma \widehat{\beta}(t)\right], \quad \widehat{\beta}(0)=0
$$

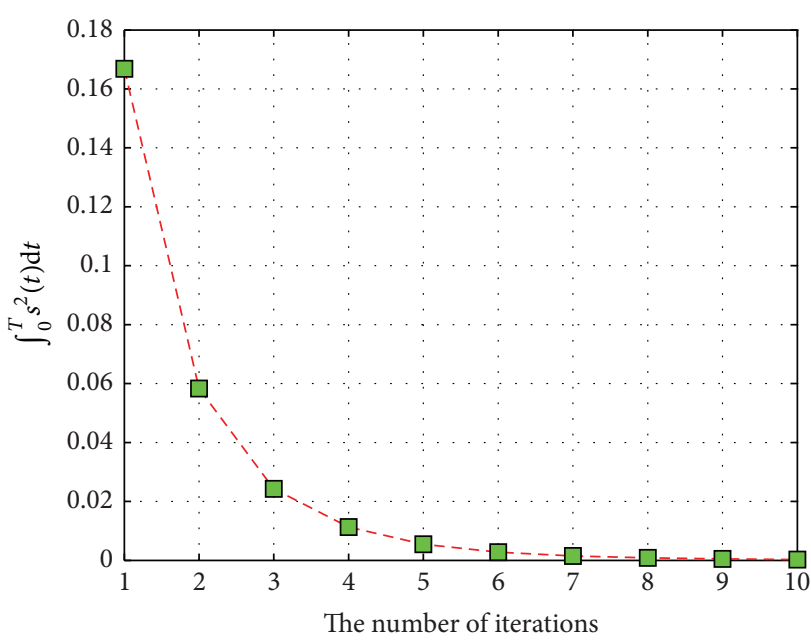

FIgURE 6: $\int_{0}^{T} s_{k}^{2}(t) \mathrm{d} t$ versus the number of iterations in Case 1.

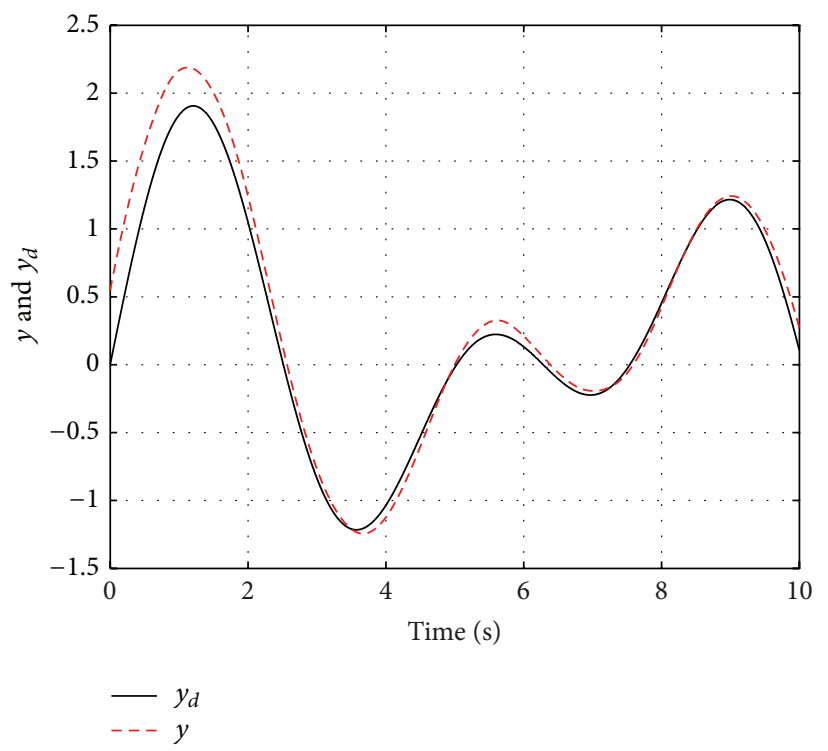

FIGURE 7: System output $y$ on $y_{d}(k=1)$ in Case 2.

The design parameters are given by $\Gamma=\operatorname{diag}\{0.01\}$ and $\sigma=0.5$. Since traditional adaptive controller does not run repeatedly, the notation $k$ in this case does not have any practical meaning. Figure 12, 13, and 14 provide simulation results. From the simulation results shown below, it is obvious that the adaptive controller cannot achieve perfect tracking performance of the system output and reference trajectory.

As observed in simulation results above, the proposed AILC can achieve a good tracking performance and tracking errors decrease along the iteration axis, which demonstrates the validity of the proposed AILC approach in this paper. 


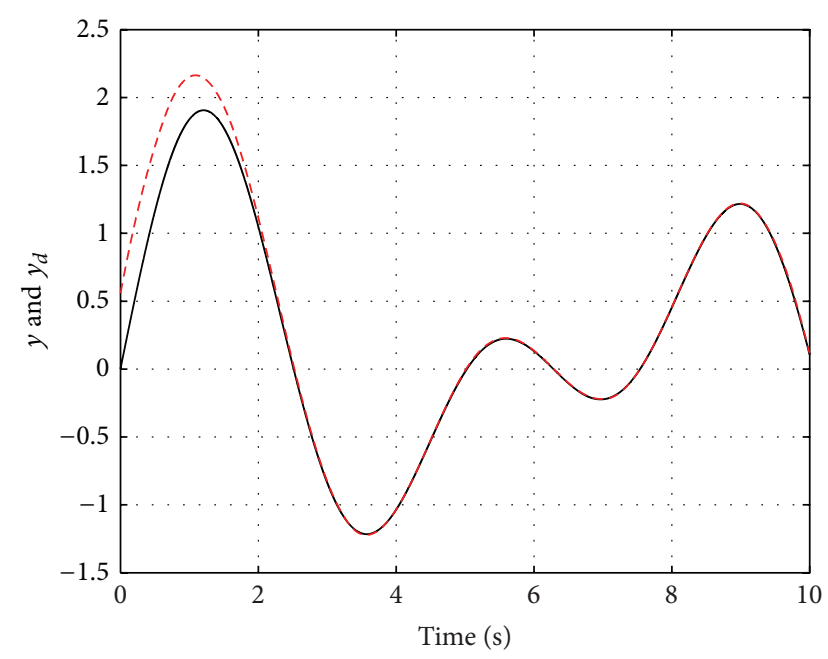

$-y_{d}$

$---y$

Figure 8: System output $y$ on $y_{d}(k=10)$ in Case 2.

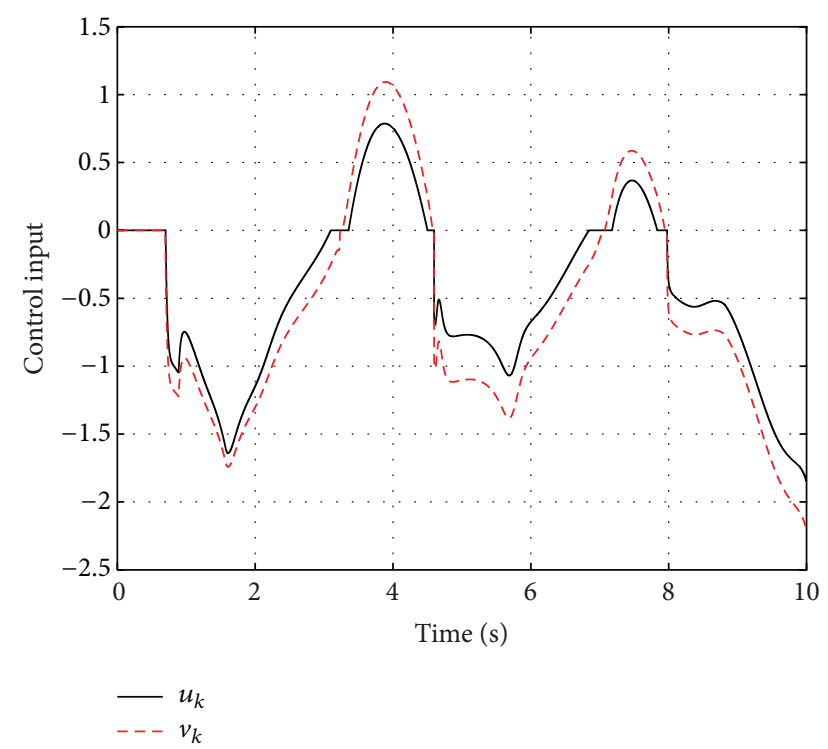

Figure 9: Control input $u_{k}$ and $v_{k}(k=1)$ in Case 2.

\section{Conclusions}

In this paper, a new AILC scheme is proposed for a class of nonlinear time-varying systems with both unknown timevarying time-delay and unknown input dead-zone nonlinearity in the presence of disturbance running on a finite time interval repetitively. A novel representation of the deadzone output is given. Using appropriate Lyapunov-Krasovskii functional in the Lyapunov function candidate, the uncertainties from unknown time-varying delays are removed such that control law is delay-independent. The identical initial condition for ILC is relaxed by introducing the boundary layer. The hyperbolic tangent function is employed to avoid the possible singularity problem. Theoretical analysis by

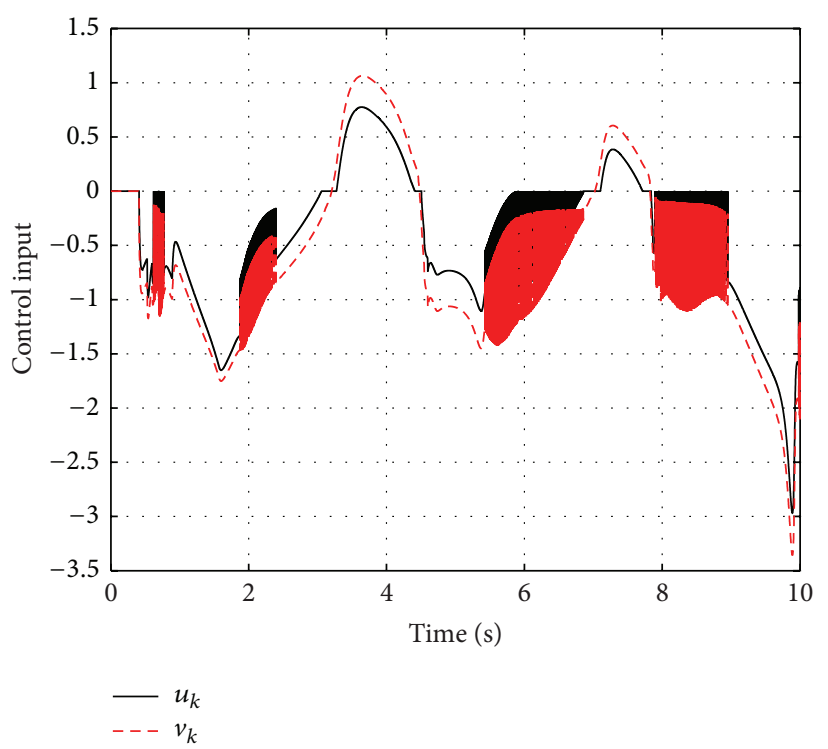

Figure 10: Control input $u_{k}$ and $v_{k}(k=10)$ in Case 2.

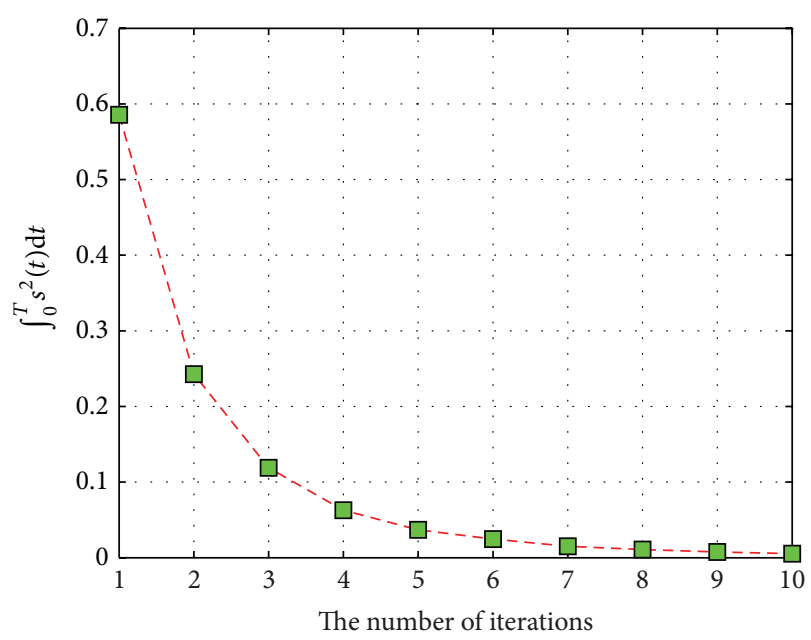

FIgURE 11: $\int_{0}^{T} s_{k}^{2}(t) \mathrm{d} t$ versus the number of iterations in Case 2.

constructing Lyapunov-like CEF has shown that the tracking errors converge to a small residual domain around the origin as iteration goes to infinity. At the same time, all the closed-loop signals remain bounded. Simulation results have been provided to demonstrate the effectiveness the proposed control scheme.

\section{Appendix}

Proof of Lemma 13. For convenience in expression, denote $x=s_{k}(t) / \eta(t)$. We rewrite inequality (32) as

$$
\frac{1}{b}<\tanh ^{2}(x)=\left(\frac{e^{x}-e^{-x}}{e^{x}+e^{-x}}\right)^{2}=1-\left(\frac{2}{e^{x}+e^{-x}}\right)^{2} .
$$




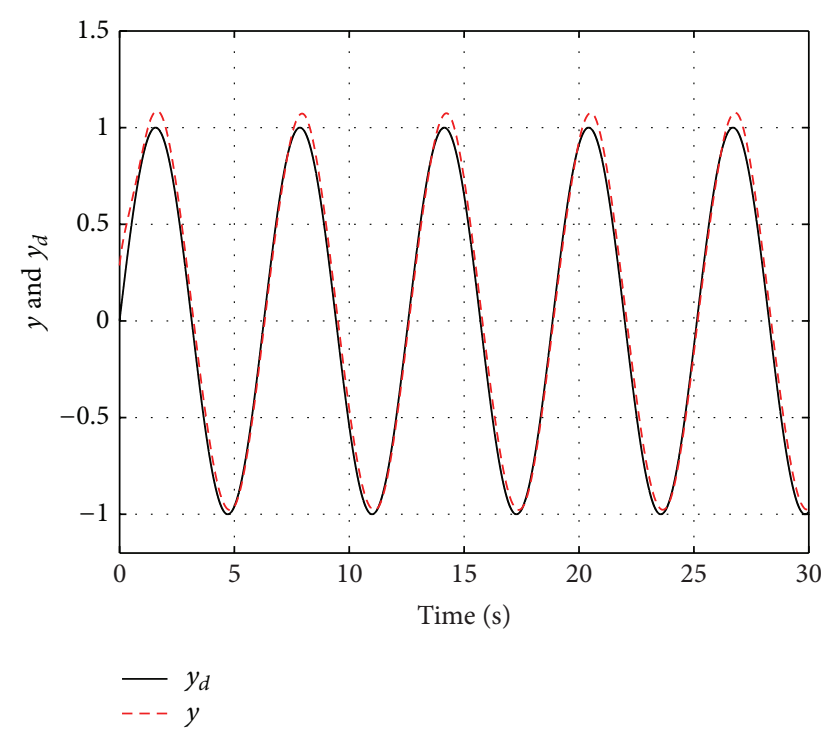

Figure 12: System output $y$ on $y_{d}$ in Case 3 .

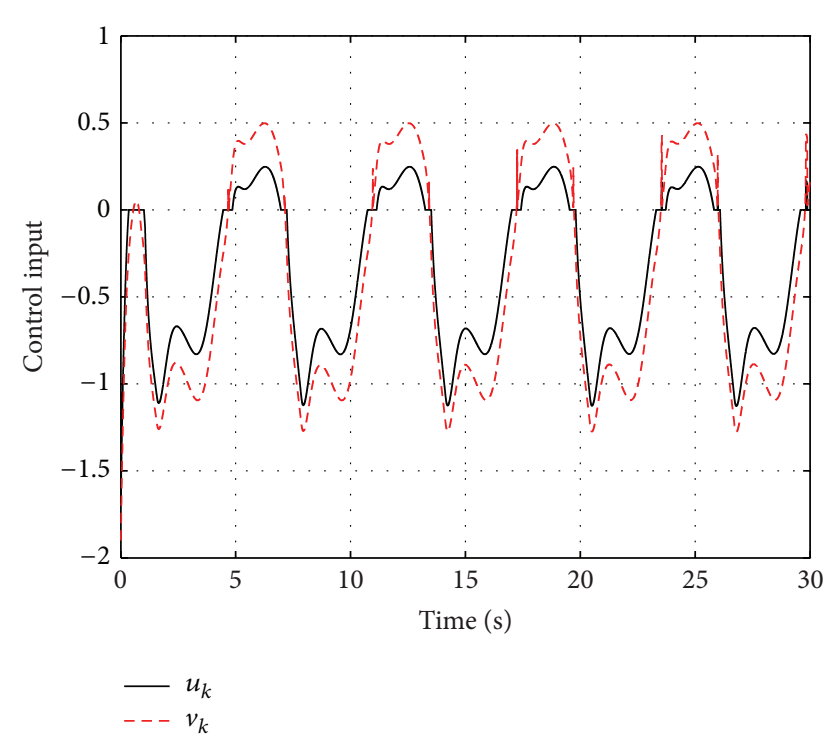

Figure 13: Control input $u_{k}$ and $v_{k}$ in Case 3.

Noting the fact that $e^{x}$ and $e^{-x}$ are positive, it follows from (A.1) that

$$
e^{x}+e^{-x}>2 \sqrt{\frac{b}{b-1}} .
$$

Then, we can obtain

$$
e^{2 x}-2 \sqrt{\frac{b}{b-1}} e^{x}+1>0
$$

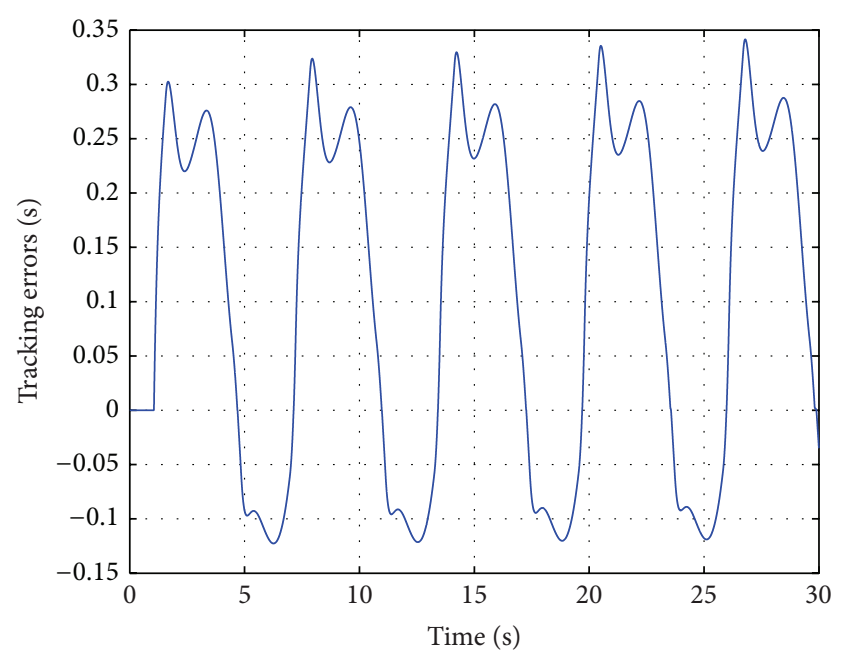

FIGURE 14: Tracking error $s(t)$ versus time.

Solving the quadratic inequality (A.3), we can have

$$
\begin{aligned}
& 0<e^{x}<\sqrt{\frac{b}{(b-1)}}-\sqrt{\frac{1}{(b-1)}} \\
& \text { or } e^{x}>\sqrt{\frac{b}{(b-1)}}+\sqrt{\frac{1}{(b-1)}} .
\end{aligned}
$$

On the other hand, from $\left|s_{k}(t)\right|>m \eta(t)$, we know that

$$
x<-m \text { or } x>m
$$

which implies

$$
\begin{aligned}
0 & <e^{x}<\frac{1}{\sqrt{b /(b-1)}+\sqrt{1 /(b-1)}} \\
& =\sqrt{\frac{b}{(b-1)}-\sqrt{\frac{1}{(b-1)}}} \\
& \text { or } e^{x}>\sqrt{\frac{b}{(b-1)}}+\sqrt{\frac{1}{(b-1)}} .
\end{aligned}
$$

Obviously, from the homology of (A.4) and (A.6), we know that Lemma 13 holds.

\section{Conflict of Interests}

The authors declare that there is no conflict of interests regarding the publication of this paper.

\section{References}

[1] S. Arimoto, S. Kawamrua, and F. Miyazaki, "Bettering operation of robots by learning," Journal of Robotic Systems, vol. 11, no. 2, pp. 123-140, 1984.

[2] S. Oh, Z. Bien, and I. H. Suh, "An iterative learning control method with application for the robot manipulator," IEEE Transactions on Robotics and Automation, vol. 4, no. 5, pp. 508$514,1988$. 
[3] T. Y. Kuc, K. Nam, and J. S. Lee, "An iterative learning control of robot manipulators," IEEE Transactions on Robotics and Automation, vol. 7, no. 6, pp. 835-841, 1991.

[4] D. H. Owens, "Universal iterative learning control using adaptive high-gain feedback," International Journal of Adaptive Control and Signal Processing, vol. 7, no. 5, pp. 383-388, 1993.

[5] T.-J. Jang, C.-H. Choi, and H.-S. Ahn, "Iterative learning control in feedback systems," Automatica, vol. 31, no. 2, pp. 243-248, 1995.

[6] C.-J. Chien and J.-S. Liu, "A P-type iterative learning controller for robust output tracking of nonlinear time-varying systems," International Journal of Control, vol. 64, no. 2, pp. 319-334, 1996.

[7] J. H. Moon, T. Y. Doh, and M. J. Chung, "A robust approach to iterative learning control design for uncertain systems," Automatica, vol. 34, no. 8, pp. 1001-1004, 1998.

[8] D. Wang, "Convergence and robustness of discrete time nonlinear systems with iterative learning control," Automatica, vol. 34, no. 11, pp. 1445-1448, 1998.

[9] S. S. Saab, "On a discrete-time stochastic learning control algorithm," IEEE Transaction on Automatic Control, vol. 46, no. 8, pp. 1333-1336, 2001.

[10] M. Sun and D. Wang, "Iterative learning control with initial rectifying action," Automatica, vol. 38, no. 7, pp. 1177-1182, 2002.

[11] H.-F. Chen and H.-T. Fang, "Output tracking for nonlinear stochastic systems by iterative learning control," IEEE Transaction on Automatic Control, vol. 49, no. 4, pp. 583-588, 2004.

[12] A. Madady, "PID type iterative learning control with optimal gains," International Journal of Control Automation and Systems, vol. 6, no. 2, pp. 194-203, 2008.

[13] M. French and E. Rogers, "Non-linear iterative learningby an adaptive Lyapunov technique," International Journal of Control, vol. 73, no. 10, pp. 840-850, 2000.

[14] J. Y. Choi and J. S. Lee, "Adaptive iterative learning control of uncertain robotic systems," IEE Proceedings-Control Theory and Application, vol. 147, no. 2, pp. 217-223, 2000.

[15] J.-X. Xu and B. Viswanathan, "Adaptive robust iterative learning control with dead zone scheme," Automatica, vol. 36, no. 1, pp. 91-99, 2000.

[16] J.-X. Xu and Y. Tan, "A composite energy function-based learning control approach for nonlinear systems with timevarying parametric uncertainties," IEEE Transactions on Automatic Control, vol. 47, no. 11, pp. 1940-1945, 2002.

[17] Y.-P. Tian and X. Yu, "Robust learning control for a class of nonlinear systems with periodic and aperiodic uncertainties," Automatica, vol. 39, no. 11, pp. 1957-1966, 2003.

[18] A. Tayebi, "Adaptive iterative learning control for robot manipulators," Automatica, vol. 40, no. 7, pp. 1195-1203, 2004.

[19] J.-X. Xu, Y. Tan, and T.-H. Lee, "Iterative learning control design based on composite energy function with input saturation," Automatica, vol. 40, no. 8, pp. 1371-1377, 2004.

[20] J. X. Xu and J. Xu, "On iterative learning from different tracking tasks in the presence of time-varying uncertainties," IEEE Transactions on Systems, Man, and Cybernetics B: Cybernetics, vol. 34, no. 1, pp. 589-597, 2004.

[21] M. X. Sun and S. S. Ge, "Adaptive repetitive control for a class of nonlinearly parametrized systems," IEEE Transaction on Automatic Control, vol. 51, no. 10, pp. 1684-1688, 2006.

[22] R. H. Chi, Z. S. Hou, and J. X. Xu, "Adaptive ILC for a class of discrete-time systems with iteration-varying trajectory and random initial condition," Automatica, vol. 44, no. 8, pp. 22072213, 2008.
[23] C. J. Chien, C. T. Hsu, and C. Y. Yao, "Fuzzy system-based adaptive iterative learning control for nonlinear plants with initial state errors," IEEE Transactions on Fuzzy Systems, vol. 12, no. 5, pp. 724-732, 2004.

[24] A. Tayebi and C.-J. Chien, "A unified adaptive iterative learning control framework for uncertain nonlinear systems," IEEE Transactions on Automatic Control, vol. 52, no. 10, pp. 1907-1913, 2007.

[25] C. J. Chien, "A combined adaptive law for fuzzy iterative learning control of nonlinear systems with varying control tasks," IEEE Transaction on Fuzzy Systems, vol. 16, no. 1, pp. 4051, 2008.

[26] Y.-C. Wang and C.-J. Chien, "Decentralized adaptive fuzzy neural iterative learning control for nonaffine nonlinear interconnected systems," Asian Journal of Control, vol. 13, no. 1, pp. 94-106, 2011.

[27] W. Chen, J. M. Li, and J. Li, "Practical adaptive iterative learning control framework based on robust adaptive approach," Asian Journal of Control, vol. 13, no. 1, pp. 85-93, 2011.

[28] S. S. Ge, F. Hong, and T. H. Tee, "Robust adaptive control of nonlinear systems with unknown time delays," Automatica, vol. 41, no. 12, pp. 1181-1190, 2005.

[29] C. C. Hua, G. Feng, and X. P. Guan, "Robust controller design of a class of nonlinear time delay systems via backstepping method," Automatica, vol. 44, no. 2, pp. 567-573, 2008.

[30] W. S. Chen, L. C. Jiao, J. Li, and R. H. Li, "Adaptive NN backstepping output-feedback control for stochastic nonlinear strictfeedback systems with time-varying delays," IEEE Transactions on Systems, Man and Cybernetics B: Cybernetics, vol. 40, no. 3, pp. 1-12, 2009.

[31] Z.-F. Liu, C.-X. Cai, and Y. Zou, "Switching signal design for exponential stability of uncertain discrete-time switched timedelay systems," Journal of Applied Mathematics, vol. 2013, Article ID 416292, 11 pages, 2013.

[32] B. Zhou, H. J. Gao, Z. L. Lin, and G.-R. Duan, "Stabilization of linear systems with distributed input delay and input saturation," Automatica, vol. 48, no. 5, pp. 712-724, 2012.

[33] B. Zhou, Z. L. Lin, and G.-R. Duan, "Truncated predictor feedback for linear systems with long time-varying input delays," Automatica, vol. 48, no. 10, pp. 2387-2399, 2012.

[34] B. Zhou, Z.-Y. Li, and Z. L. Lin, "Observer based output feedback control of linear systems with input and output delays," Automatica, vol. 49, no. 7, pp. 2039-2052, 2013.

[35] B. Zhou, Truncated Predictor Feedback for Time-Delay Systems, Springer, Berlin, Germany, 2014.

[36] Y. Chen, Z. Gong, and C. Wen, "Analysis of a high-order iterative learning control algorithm for uncertain nonlinear systems with state delays," Automatica, vol. 34, no. 3, pp. 345353, 1998 .

[37] K. H. Prak, Z. Bien, and D. H. Hwang, "Design of an iterative learning controller for a class of linear dynamic systems with time delay," IEE Proceedings-Control Theory and Applications, vol. 145, no. 6, pp. 507-512, 1998.

[38] M. X. Sun and D. W. Wang, "Initial condition issues on iterative learning control for non-linear systems with time delay," International Journal of Systems Science, vol. 32, no. 11, pp. 1365-1375, 2001.

[39] W. S. Chen, "Adaptive learning control of linear systems with completely unknown time delays," International Journal of Automation and Computing, vol. 6, no. 2, pp. 177-185, 2009. 
[40] W. S. Chen and L. Zhang, "Adaptive iterative learning control for nonlinearly parameterized systems with unknown timevarying delays," International Journal of Control, Automation and Systems, vol. 8, no. 2, pp. 177-186, 2010.

[41] W. S. Chen, Y. L. Wang, and J. M. Li, "Adaptive learning control for nonlinearly parameterized systems with periodically timevarying delays," Acta Automatica Sinica, vol. 34, no. 12, pp. 15561560, 2008.

[42] G. Tao and P. V. Kokotović, "Adaptive control of plants with unknown dead-zones," IEEE Transactions on Automatic Control, vol. 39, no. 1, pp. 59-68, 1994.

[43] X. S. Wang, H. Hong, and C. Y. Su, "Model reference adaptive control of continuous-time systems with an unknown input dead-zone," IEE Proceedings-Control Theory and Applications, vol. 150, no. 3, pp. 261-266, 2003.

[44] X. D. Tang, G. Tao, and M. J. Suresh, "Adaptive actuator failure compensation for parametric strict feedback systems and an aircraft application," Automatica, vol. 39, no. 11, pp. 1975-1982, 2003.

[45] X.-S. Wang, C.-Y. Su, and H. Hong, "Robust adaptive control of a class of nonlinear systems with unknown dead-zone," Automatica, vol. 40, no. 3, pp. 407-413, 2004.

[46] T. P. Zhang and S. S. Ge, "Adaptive neural control of MIMO nonlinear state time-varying delay systems with unknown dead-zones and gain signs," Automatica, vol. 43, no. 6, pp. 10211033, 2007.

[47] T. P. Zhang and S. S. Ge, "Adaptive dynamic surface control of nonlinear systems with unknown dead zone in pure feedback form," Automatica, vol. 44, no. 7, pp. 1895-1903, 2008.

[48] J.-X. Xu, J. Xu, and T. H. Lee, "Iterative learning control for systems with input deadzone," IEEE Transactions on Automatic Control, vol. 50, no. 9, pp. 1455-1459, 2005.

[49] S. S. Ge and K. P. Tee, "Approximation-based control of nonlinear MIMO time-delay systems," Automatica, vol. 43, no. 1, pp. 31-43, 2007.

[50] P. A. Ioannou and J. Sun, Robust Adaptive Control, PrenticeHall, Englewood Cliffs, NJ, USA, 1995. 


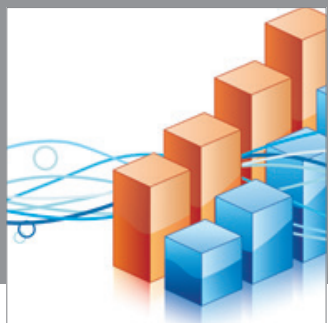

Advances in

Operations Research

mansans

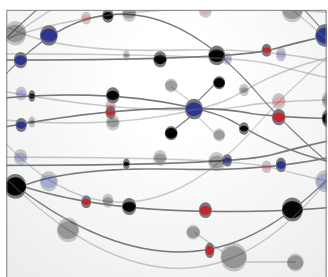

The Scientific World Journal
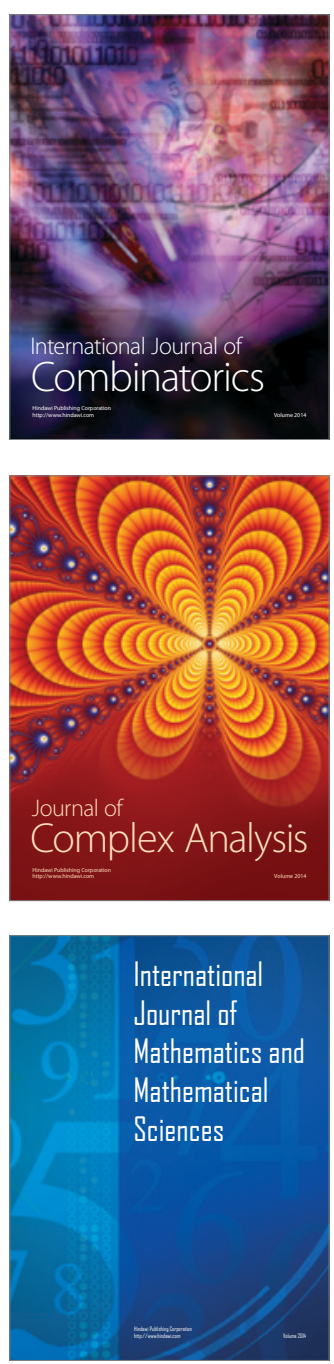
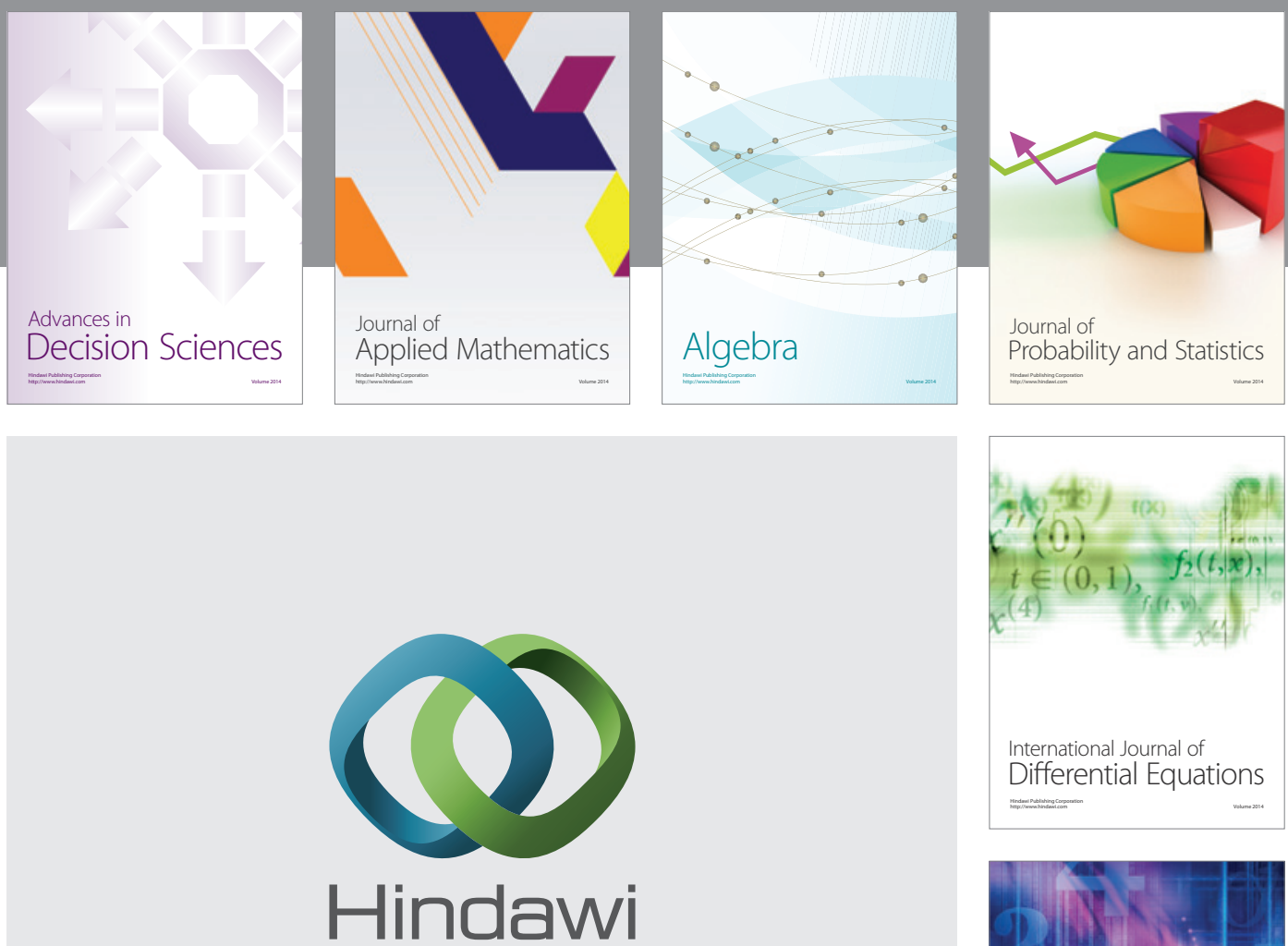

Submit your manuscripts at http://www.hindawi.com
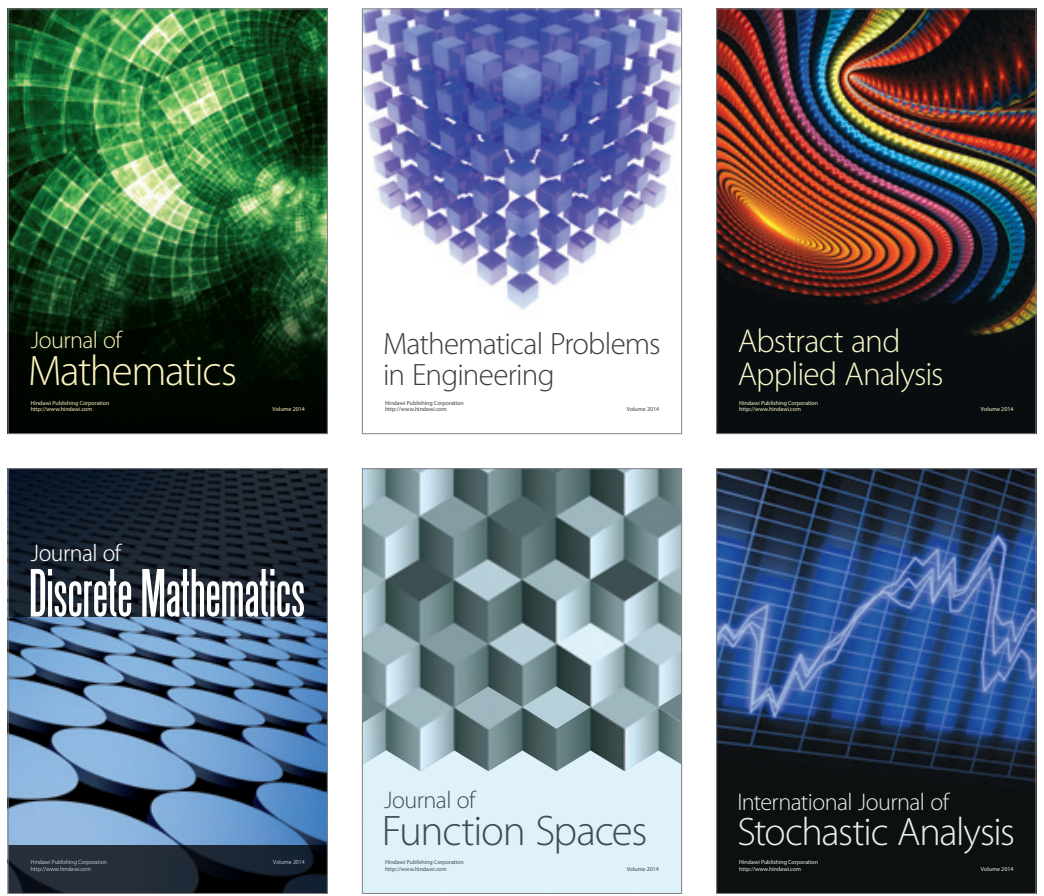

Journal of

Function Spaces

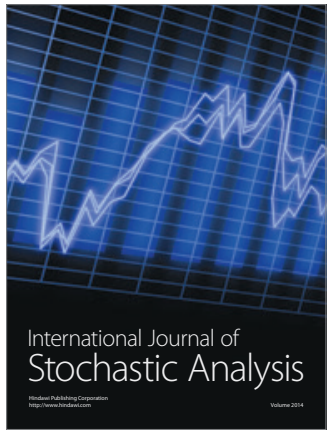

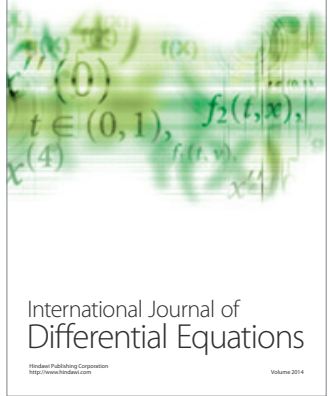
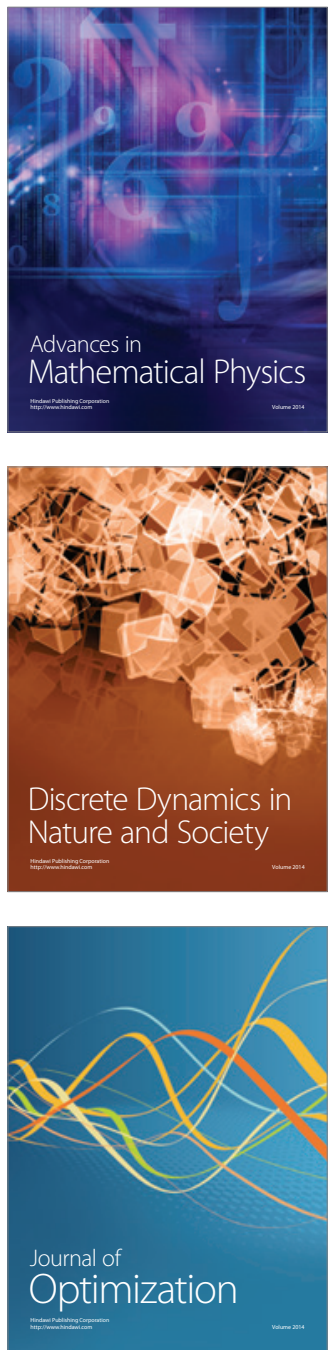